\title{
Postsynaptic ERG Potassium Channels Limit Muscle Excitability to Allow Distinct Egg-Laying Behavior States in Caenorhabditis elegans
}

\author{
Kevin M. Collins and Michael R. Koelle \\ Department of Molecular Biophysics and Biochemistry, Yale University, New Haven, Connecticut 06520-8024
}

Caenorhabditis elegans regulates egg laying by alternating between an inactive phase and a serotonin-triggered active phase. We found that the conserved ERG [ether-a-go-go (EAG) related gene] potassium channel UNC-103 enables this two-state behavior by limiting excitability of the egg-laying muscles. Using both high-speed video recording and calcium imaging of egg-laying muscles in behaving animals, we found that the muscles appear to be excited at a particular phase of each locomotor body bend. During the inactive phase, this rhythmic excitation infrequently evokes calcium transients or contraction of the egg-laying muscles. During the serotonin-triggered active phase, however, these muscles are more excitable and each body bend is accompanied by a calcium transient that drives twitching or full contraction of the egg-laying muscles. We found that ERG-null mutants lay eggs too frequently, and that ERG function is necessary and sufficient in the egg-laying muscles to limit egg laying. ERG K ${ }^{+}$channels localize to postsynaptic sites in the egg-laying muscle, and mutants lacking ERG have more frequent calcium transients and contractions of the egg-laying muscles even during the inactive phase. Thus ERG channels set postsynaptic excitability at a threshold so that further adjustments of excitability by serotonin generate two distinct behavioral states.

\section{Introduction}

Changes in synaptic strength enable animals to enter different behavioral states and underlie various forms of learning and memory. Synaptic plasticity is regulated by mechanisms that function on different timescales, from seconds to years. Neurotransmitter signaling through G-protein-coupled receptors affects cell and synaptic excitability within seconds via regulation of plasma membrane and intracellular ion channels (McDonald et al., 1994). This kind of modulatory signaling alters neural circuit activity and behavior (Marder and Bucher, 2007). The resulting short-term changes in synaptic $\mathrm{Ca}^{2+}$ signaling regulate more stable forms of synaptic plasticity, including long-term potentiation or long-term depression (Lüscher et al., 2000). Dissecting the molecular basis of synaptic excitability and how it is regulated by fast and slow plasticity is critical to understanding behavior. Be-

\footnotetext{
Received Aug. 15, 2012; revised 0ct. 17, 2012; accepted Nov. 14, 2012.

Author contributions: K.M.C. and M.R.K. designed research; K.M.C. performed research; K.M.C. and M.R.K. analyzed data; K.M.C. and M.R.K. wrote the paper.

This work was funded by a grant to M.R.K. from the National Institute of Neurological Disorders and Stroke (NS036918) and by fellowships to K.M.C. from the National Institutes of Health (GM079813) and the American Heart Association (POST4990016). Confocal instrumentation was supported by a grant to the Yale Liver Center (DK34989). We are grateful to Amy Bany, Andy Bellemer, and Antony Jose for important preliminary work; Rene Garcia, Bob Horvitz, Liqun Luo, Kang Shen, and Paul Sternberg for reagents and discussions; and Sloan Warren, Tony Koleske, and members of the Koelle laboratory for insightful comments. Some strains used were obtained from the Caenorhabditis Genetics Center.

The authors declare no competing financial interests.

Correspondence should be addressed to Michael Koelle, Department of Molecular Biophysics and Biochemistry, Yale University School of Medicine, 333 Cedar Street, SHM CE30, P.0. Box 208024, New Haven, CT 06520-8024. E-mail: michael.koelle@yale.edu.

DOI:10.1523/JNEUROSCI.3896-12.2013

Copyright $\odot 2013$ the authors $\quad 0270-6474 / 13 / 330761-15 \$ 15.00 / 0$
}

cause most studies focus on synaptic connections within complex neural circuits, the precise relationships between molecules, synaptic excitability, and behavioral output in vivo remain poorly understood.

Caenorhabditis elegans egg-laying behavior offers many experimental advantages for studying the molecular basis of synaptic activity and its modulation during distinct behavior states. Egg laying is controlled by a simple circuit in which eight motor neurons, which themselves receive little synaptic input, form a large synapse onto a set of vulval muscles and stimulate their contraction. Egg laying involves alternating between two distinct behavioral states: quiescent periods of about $20 \mathrm{~min}$, during which no egg laying occurs, and egg-laying active phases lasting several minutes. The active phases appear to result when two of the motor neurons release serotonin to act via G-protein-coupled receptors on the vulval muscles and increase their excitability (Waggoner et al., 1998; Hapiak et al., 2009). Strong regulation is superimposed on the pattern of alternating egglaying behavioral states. For example, worms virtually halt egg laying in the absence of food and rapidly restart the behavior when refed (Dong et al., 2000). Egg laying has been studied genetically for decades, and dozens of genes have been identified by mutations that either block (Desai et al., 1988) or increase egg laying (Bany et al., 2003). However, the activity of the underlying egg-laying motor circuit and how it is modulated by serotonin have not been well defined. The ability to leverage egg-laying mutants to understand the molecular mechanisms that regulate synaptic function has been limited by the tools available to analyze the egg-laying synapse and its behavioral output.

In this work, we developed new imaging and behavioral assays that enabled us to analyze the structure, activity, and behavioral 
output of the egg-laying synapse. We used these tools to determine how the ERG [ether-a-go-go (EAG) related gene] $\mathrm{K}^{+}$channel functions at the egg-laying synapse to allow serotonin to generate distinct active and inactive behavioral states for egg laying.

\section{Materials and Methods}

Nematode culture, strains, and maintenance. C. elegans strains were maintained as hermaphrodites at $20^{\circ} \mathrm{C}$ on nematode growth medium (NGM) agar plates with Escherichia coli OP50 as a source of food as described previously (Brenner, 1974). All strains are derived from the Bristol N2 wild-type strain, and all assays were performed using age-matched adult hermaphrodites $24-40 \mathrm{~h}$ past the late L4 stage. unc-103 alleles were isolated via ethyl methanesulfonate mutagenesis (Bany et al., 2003; Bellemer et al., 2011): vs39 (C507Y), vs157 (S607fs), vs158 (S302F).

Molecular biology and transgenes. Transgenes were generated by microinjection of plasmid DNA into hermaphrodites. For expression of wild-type ERG in all vulval and body-wall muscles, a cDNA for the vulval muscle-expressed isoform, unc-103e (Reiner et al., 2006), was inserted into pPD96.52 (1999 Fire Lab Vector kit) between the myo-3 promoter and unc-54 3' UTR to generate plasmid pKMC134. This plasmid, or the empty vector control, were coinjected with a plasmid expressing GFP in muscle (pAB25; Bellemer et al., 2011) at $5 \mathrm{ng} / \mu \mathrm{l}$ each along with the lin-15 rescuing plasmid pL15EK (Clark et al., 1994) (50 ng/ $\mu \mathrm{l}$ ) into LX1476 unc-103(sy673, e1597dm); lin-15(n765ts) animals. For reexpression in the hermaphrodite-specific neuron (HSN), coding sequences for the HSN-expressed ERG isoform, unc-103f (Garcia and Sternberg, 2003; Reiner et al., 2006), were inserted into the empty vector pJM66A (Moresco and Koelle, 2004) between the tph-1 promoter and unc-54 3' UTR to generate pKMC112. To help increase expression levels to ensure that any failure to observe phenotypic rescue was not due to weak expression from the $t p h-1$ promoter, introns 5-10 were retained in the unc-103f coding sequence (see below). Similar results were seen with the unc-103f cDNA bearing no introns (data not shown). pKMC112 (ERG) or the corresponding empty vector (pJM66A) were coinjected with a plasmid expressing GFP in the HSN (pJM60A; Moresco and Koelle, 2004) at $80 \mathrm{ng} / \mu \mathrm{l}$ each along with the L15EK rescue plasmid (50 $\mathrm{ng} / \mu \mathrm{l}$ ) into LX1476 animals. Egg-laying behavior was assayed in five independent lines per transgene.

Tissue-specific expression of dominant-negative ERG and EAG channels. The following two complementary oligonucleotides were used to mutate sequences encoding the GFG residues in the ERG $\mathrm{K}^{+}$selectivity filter to AAA by QuikChange mutagenesis: $5^{\prime}$-CA TTA TCC ACA ATT ACA TCT ATC GCG GCC GCT AAT GTA TCG GCG ACG ACA G-3' and 5'-C TGT CGT CGC CGA TAC ATT AGC GGC CGC GAT AGA TGT AAT TGT GGA TAA TG-3'. For expression of dominant-negative ERG in muscles, pKMC134 was mutagenized to generate pKMC140 (DN-ERG). For expression in the HSN, pKMC112 was mutated to generate pKMC117 (HSN-DN-ERG). Correct mutagenesis was confirmed using the introduced NotI restriction site (italics) and DNA sequencing. To generate a dominant-negative EAG, the egl-2 cDNA was first amplified from pLR120 (LeBoeuf et al., 2007) and inserted into the muscle expression vector pPD96.52 to generate $\mathrm{pKMC186}$. The following two complementary oligonucleotides were used to mutate sequences encoding the GFG residues in the EAG $\mathrm{K}^{+}$selectivity filter to AAA by QuikChange mutagenesis: 5' -ATG AGT TGT ATG TCG ACA GTT GCG GCC GCT AAC ATT GCT AGT AAT ACA GAC- $3^{\prime}$ and $5^{\prime}-\mathrm{G}$ TCT GTA TTA CTA GCA ATG TTA GCG GCC GCA ACT GTC GAC ATA CAA CTC AT-3'. For expression of dominant-negative EAG in muscles, pKMC186 was mutated to generate pKMC214. As above, mutagenesis was confirmed using the introduced NotI restriction site (italics) and DNA sequencing. For expression in muscles, pPD96.52 (empty vector control), pKMC140 (DN-ERG), or pKMC186 (DN-EAG) were injected at $2 \mathrm{ng} / \mu \mathrm{l}$ with pAB25A (4 ng/ $\mu \mathrm{l}$; expresses GFP in muscles) and the lin-15 rescuing plasmid L15EK rescue plasmid (50 ng/ $\mu \mathrm{l}$ ) into MT8189 lin-15(n765ts) animals. For expression in the HSN, pJM66A (empty vector control) or pKMC117 (HSN-DN-ERG) were injected with pJM60A (expresses GFP in HSN) at $80 \mathrm{ng} / \mu \mathrm{l}$ each along with the L15EK rescue plasmid (50 ng/ $\mu \mathrm{l}$ ) into MT8189 animals. Egg-laying behavior was assayed in five independent lines per transgene.

Vulval muscle-specific fluorescent reporter transgenes. A $\sim 2.6 \mathrm{~kb}$ DNA fragment upstream of the unc-103e start site was amplified from genomic DNA by PCR with the following oligonucleotides: $5^{\prime}$-GGA ACTAGT GCATGC CTA TTT TAT ATT TAC AAT ATT TTA G-3' and 5'-TCA GCG CCCGGG ACC ACC ACC ACC ACA ACC-3'. This DNA fragment was ligated into pPD49.26 bearing the unc-54 3'UTR (Mello and Fire, $1995)$ to generate $\mathrm{pKMC189}$. Into this vector, coding sequences for GFP (Moresco and Koelle, 2004), GCaMP3 (Tian et al., 2009), mCherry (McNally et al., 2006), or mCD8 (Lee and Luo, 1999) fused to mCherry were inserted to generate pKMC188, pKMC265, pKMC257, and pKMC191, respectively. To generate the vulval muscle TM-mCherry reporter, pKMC191 (mCD8-mCherry, $5 \mathrm{ng} / \mu \mathrm{l}$ ) was injected along with the L15EK rescue plasmid $(50 \mathrm{ng} / \mu \mathrm{l})$ into MT8189 animals. The reporter transgene was integrated into chromosomes using UV/trimethylpsoralen, and four independent integrants (vsIs146-vsIs149) were recovered and backcrossed to the wild-type six times. Other than expression level, there were no apparent differences among the integrants in the pattern of TMmCherry expression. For double labeling of the vulval muscles with the HSN and ventral cord type C (VC) motor neurons, LX1693 animals carrying vsIs 147 were crossed with LX693 animals carrying the vsIs44 transgene, which expresses GFP from the tph-1 promoter (Tanis et al., 2008) to generate LX1847 animals. For double labeling of the vulval muscles with the VC presynaptic terminus, LX1695 animals carrying vsIs149 were crossed with TV7979 animals carrying the wyIs222 transgene (Spilker et al., 2012), which expresses GFP::RAB-3 from the unc-4 promoter to generate LX1865 animals. To construct the vulval muscle calcium reporter, pKMC265 (GCaMP3, $20 \mathrm{ng} / \mu \mathrm{l})$ and pKMC257 (mCherry, $2 \mathrm{ng} / \mu \mathrm{l}$ ) were injected along with the L15EK rescue plasmid $(50 \mathrm{ng} / \mu \mathrm{l})$ into MT8189 animals. The GCaMP3 reporter transgene was integrated as above, and four independent integrants (vsIs150-vsIs153) were recovered and backcrossed to the wild type six times. There were no detectable differences in expression pattern among the integrants. LX1776 carrying the $v$ sIs 153 transgene had the lowest level of expression and no apparent phenotypic abnormalities. To reduce blue-light sensitivity during imaging, KG1180 animals carrying the lite-1(ce314) mutation (Edwards et al., 2008) were crossed to LX1776 animals to generate LX1848 vsIs153; lite-1 (ce314) animals. To move the vsIs153 reporter into the ERG W85stop-null mutant background, LX1442 animals were mated with KG1180 animals and the cross progeny were crossed with LX1848 animals to generate LX1849 vsIs153; unc-103(sy673, e1597dm); lite1(ce314) animals. A control transgene (vsEx733) expressing GFP and $\mathrm{mCherry}$ in the vulval muscles from the unc-103e promoter was prepared by injecting pKMC188 (GFP, $2 \mathrm{ng} / \mu \mathrm{l}$ ) and pKMC257 (mCherry, $2 \mathrm{ng} /$ $\mu \mathrm{l})$ along with L15EK rescue plasmid into LX1832 lite-1(ce314), lin15(n765ts) animals to generate LX1901.

Single-copy MosSCI transgenes. The unc-103 3' UTR region was amplified from genomic DNA by PCR using the following oligonucleotides: 5'-TGA CTCGAG GGTACC ACGCGT CCC GGT TTC TCT CTT TCT CTG- $3^{\prime}$ and $5^{\prime}$-CGC CTTAAG GCC TTT TTG CAC TGT TGA GTG-3'. The amplified 3'UTR and the vulval muscle-specific unc-103e promoter (above) were inserted into pCFJ151 (Frøkjaer-Jensen et al., 2008) to generate the empty vulval muscle-specific MosSCI vector pKMC176. To ensure robust ERG::GFP expression from single-copy transgenes, an unc-103e genomic cDNA/genomic DNA hybrid was first constructed in pUC19. Briefly, unc-103 exons 5-11 were PCR-amplified from worm genomic DNA and inserted into the unc-103e cDNA at unique BstXI and AccI sites to generate $\mathrm{pKMC100.} \mathrm{To} \mathrm{make} \mathrm{a} \mathrm{functional} \mathrm{ERG-GFP} \mathrm{fusion,}$ a NotI site was inserted into pKMC100 near the $3^{\prime}$ end of unc-103e in a region encoding five consecutive glycine residues by QuikChange mutagenesis using the following complementary oligonucleotides to generate pKMC178: 5' -CGT CTT CCG AAT GGA GGT GGT GCG GCC GCT GGA GGT GGT GTT GTC GAT GAG ATG AGA GTC- ${ }^{\prime}$ and 5' $^{\prime}$-GAC TCT CAT CTC ATC GAC AAC ACC ACC TCC AGC GGC CGC ACC ACC TCC ATT CGG AAG ACG-3'. Coding sequences for GFP were then inserted at the NotI site in pKMC178 to generate pKMC179. Wild-type ERG::GFP was amplified from pKMC179 by PCR and inserted into the MoSCI vector $\mathrm{pKMC176}$ to generate $\mathrm{pKMC180.}$. Coding sequences for 
A
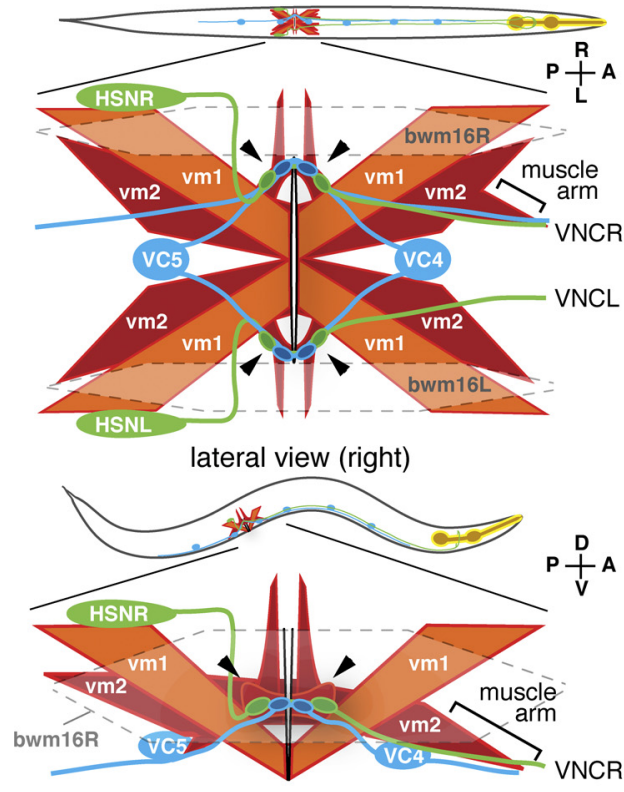
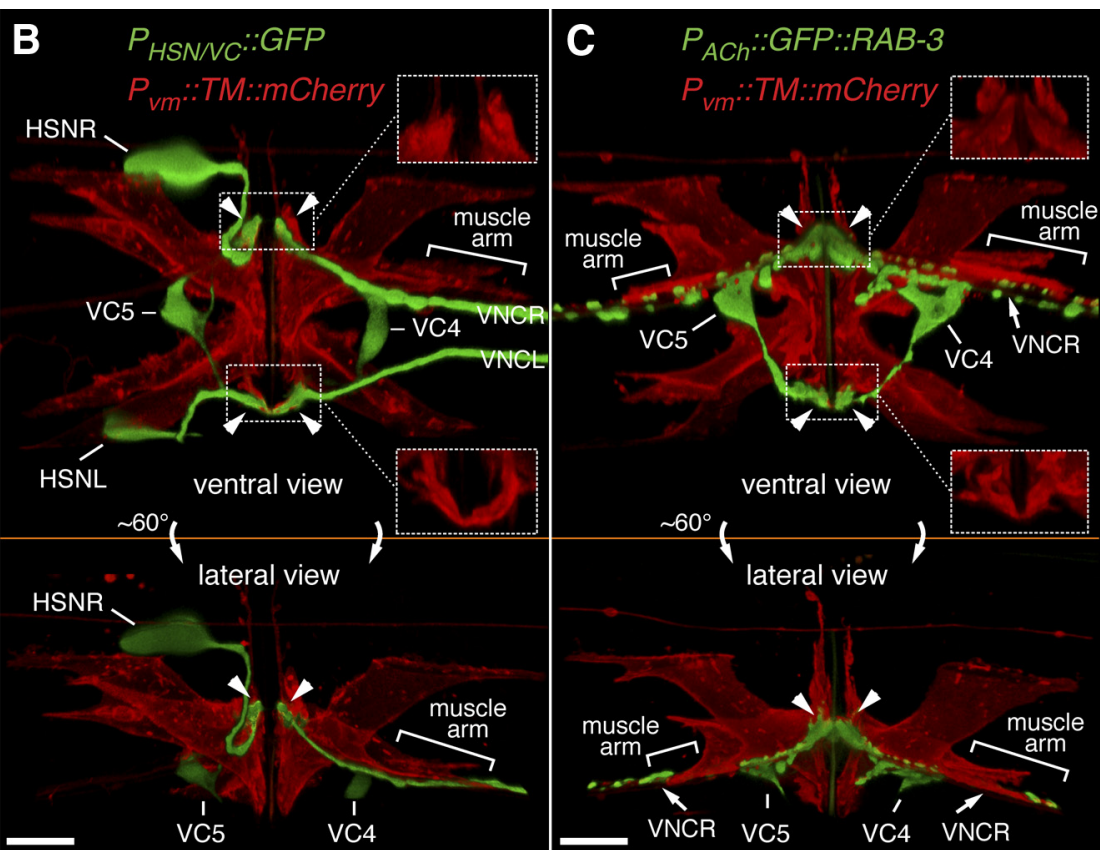

Figure 1. Anatomy of the egg-laying system. A, Diagrams showing ventral (top) or lateral (bottom) views of C. elegans and expanded views of the egg-laying system. HSN (green) and VC (blue) motor neurons as well as vm1 (orange) and vm2 (red) vulval muscles are shown. Six VC neurons send processes along the right ventral nerve cord (VNC). VC 4 and VC5 have cell bodies close to the vulva and extend processes bilaterally to the vm2s. vm2 cells extend arms laterally onto which HSN and VC presynaptic termini form (arrowheads). vm2 cells on the right side also extend separate muscle arms (brackets), which receive additional synapses from the right ventral nerve cord. Gap junctions electrically connect vm cells, but anterior and posterior vm cells appear to be isolated from each other (solid black lines) but postsynaptic to the same neurons. The vulval muscles fill a gap between body-wall muscle cells bwm16L/R (dashed line). Axes indicating anterior ( $A$ ), posterior ( $P$ ), left $(\mathrm{L})$, right $(\mathrm{R})$, dorsal $(\mathrm{D})$, and ventral $(\mathrm{V})$ are shown. Micrographs and schematics in this work are often right lateral views to allow visualization of the synapses between vm2 and the right ventral nerve cord. $\boldsymbol{B}$, Presynaptic motor neurons interact with the vulval muscle at a large postsynaptic terminus. Transgenic worms expressed mCherry fused to a transmembrane domain (TM::mCherry) at the plasma membrane from a vulval muscle-specific promoter $\left(P_{v m}\right)$ and expressed GFP from a second promoter strongly in the HSN and weakly in the VC4 and VC5 neurons. Arrowheads indicate presynaptic varicosities, and regions indicated with dotted boxes are magnified at right corners (150\%) with only TM::mCherry labeling to reveal the vulval muscle postsynaptic termini onto which the HSN and VCs make synaptic contact. Scale bar, $10 \mu \mathrm{m}$. C, Same as $\boldsymbol{B}$ except that the GFP label is the presynaptic marker GFP::RAB-3 expressed in all cholinergic neurons. This marker fills out the VC4 and VC5 cell bodies and labels their presynaptic termini onto the vm2 lateral projections (arrowheads). It also produces punctate labeling of additional VNC synapses, and these may include the synapses made between VC neurons by VCs onto vm2 muscle arms (brackets) and by other ventral nerve cord motor neurons onto vm1 cells (White et al., 1986 ). Scale bar, $10 \mu \mathrm{m}$.

the ERG C-terminal PDZ-interaction motif (-DTIL) were replaced by a stop codon (ERG::GFP $\triangle$ PDZ) to generate pKMC204. To isolate C. elegans strains carrying single-copy ERG-GFP inserts, the MosSCI plasmids pKMC180 or pKMC204 (50 ng/ $\mu \mathrm{l})$ were injected into EG4322 animals bearing the $t t$ Ti5605 Mos1 transposon as described previously (Frøkjaer-Jensen et al., 2008). Correct replacement of the Mos1 transposon by the single-copy transgene ( $v$ sSil, wild-type ERG::GFP; $v s S i 11$, ERG::GFP $\triangle$ PDZ) was confirmed by DNA sequencing and expression of GFP. To check for phenotypic rescue, animals bearing vsSil or vsSill transgenes were crossed with LX1442 ERG-null mutants (W85Stop) to generate strains LX1678 and LX1685, respectively. For double labeling of MosSCI transgenes with the vulval muscle TM-mCherry reporter, vsIs149 was crossed into LX1678 and LX1685 animals.

Behavior assays and video recording. Unlaid eggs and early-stage eggs that were laid were quantitated as described using age-matched adult hermaphrodites 24-40 h past the late L4 stage (Chase and Koelle, 2004). For bright-field recording of behaving animals, a Casio EX-FC150 camera was mounted onto an eye tube of a Zeiss Axiovert 100 using Unilink and Linkarm adaptors (Brunel Microscopes). Animals were followed by manually moving a gliding stage. To measure intervals between egglaying events (see Fig. 5), single hermaphrodite animals were picked to NGM plates seeded with $30 \mu \mathrm{l}$ of an OP50 bacterial culture. Video recordings were performed through a $10 \times$ objective at 30 frames per second $(\mathrm{fps})$ at $640 \times 480$ pixels. Videos were analyzed for timing of egg-laying events and distance to adjacent smooth contractions and relaxations using ImageJ. To measure vulval muscle-twitching behavior and $\mathrm{Ca}^{2+}$ transients in behaving animals, a small amount of OP50 bacteria was scooped onto a platinum wire and used to pick one worm to an unseeded NGM plate. A $\sim 20 \times 20 \mathrm{~mm}$ chunk was immediately taken from the plate and placed worm-side down onto a $24 \times 60 \mathrm{~mm} \# 1$ coverslip and overlaid with a $22 \times 22 \mathrm{~mm} \# 1$ coverslip. The worms were allowed to recover for $1-2 \mathrm{~h}$ in a humidified chamber at $20^{\circ} \mathrm{C}$, by which time egg-laying behavior was observed to be similar to that observed in unmounted animals. Bright-field videos were recorded through a $40 \times$ Nomarski objective at $120 \mathrm{~Hz}$ at $640 \times 480$ pixels. Timing of smooth contractions, relaxations, twitches, and egg-laying events were recorded from videos using Etho Timer (Liu and Thomas, 1994). Statistical analyses for all data were performed using JMP version 9.0 (SAS Institute) or Prism 9.0, and error bars indicate 95\% confidence intervals.

Confocal microscopy and ratiometric imaging. To visualize the egglaying system and subcellular localization of ERG, age-matched adult hermaphrodite worms expressing fluorescent reporter transgenes were immobilized using $10 \mathrm{~mm}$ muscimol on 3\% agarose pads and covered with \#1 coverslips. Two-channel confocal $z$-stacks with a pinhole size of 1 Airy unit (0.9- $\mu \mathrm{m}$-thick optical sections, 12 bit images) were obtained with a Zeiss 710 Duo confocal microscope with a $63 \times$ Water C-Apochromat objective [1.2 numerical aperture (NA)] using the LSM head. For $\mathrm{Ca}^{2+}$ imaging in behaving animals, single two-channel confocal slices (18 $\mu \mathrm{m}$ thick) were collected through a $20 \times$ Plan-Apochromat objective (0.8 NA) using the 710 Duo LIVE head. Short-term (1 min) recordings were collected at $30 \mathrm{fps}$ at $512 \times 512$ pixel, 12 bit resolution while longer-term ( $6 \mathrm{~min})$ recordings were collected at $20 \mathrm{fps}$ at $256 \times$ 256 pixel, 16 bit resolution. The stage and focus were adjusted manually to keep the egg-laying system in view and focused during recording periods. Ratiometric analysis for both $z$-stacks and $\mathrm{Ca}^{2+}$ recordings was performed in Volocity (version 5, PerkinElmer). Briefly, separate ratioand-intensity-modulated ratio channels were calculated from GCaMP3 (or GFP) and mCherry fluorescence channels. Voxels with mCherry fluorescence intensities 2 SDs above background were selected as objects for measurement. Intensity-modulated images show false colors represent- 
ing the GCaMP $3 /$ mCherry or GFP/mCherry ratio displayed at the brightness of the corresponding mCherry voxels. $\mathrm{Ca}^{2+}$ transients were identified by visual inspection of ratio traces. For $1 \mathrm{~min}$ recordings, the prior local minimum in GCaMP3/mCherry ratio was used to establish a baseline for $\Delta R / R$ determination. For $6 \mathrm{~min}$ recordings, the data were first smoothed using a $150 \mathrm{~ms}$ (3 time-point) rolling average, and the lowest $10 \%$ of the GCaMP3/mCherry ratio values were averaged to establish a $\Delta R / R$ baseline. To study synapsespecific features in $z$-stacks (see Fig. 4), a $12 \mu \mathrm{m}$ $(x) \times 10 \mu \mathrm{m}(y) \times 6 \mu \mathrm{m}(z)$ box was manually placed on the synaptic region and measurements of fluorescence within this synaptic region were obtained as described above.

\section{Results}

Anatomy of the egg-laying synapse

We developed tools to visualize the egglaying system. Eight vulval muscle (vm) cells, four each of the vm1 and vm 2 class, contract to open the vulva and release eggs, and two types of motor neurons synapse onto these muscles (Fig. 1) (White et al., 1986). The VC motor neurons release acetylcholine (ACh) (Duerr et al., 2001), which activates nicotinic ACh receptors (nAChRs) expressed on the vulval muscles to drive contraction and egg laying (Waggoner et al., 2000; Kim et al., 2001). The HSNs are motor neurons that release serotonin to put the vulval muscles in a more excitable state (Weinshenker et al., 1995; Shyn et al., 2003). To visualize both sides of the egg-laying synapses, we expressed a membrane-localized mCherry protein in all eight vulval muscles to label the surface of these cells, and simultaneously expressed GFP in the HSN and VC neurons (Fig. $1 B$ ). We found that each vm2 cell extends a dendrite-like postsynaptic process laterally that meets and apposes the HSN and VC presynaptic release sites (Fig. $1 B$, arrowheads and inset boxes). Synaptic contacts form over $\sim 10$ $\mu \mathrm{m}$ on both the left and right sides of the animal in what may be the largest synapses in C. elegans. By expressing the presynaptic marker GFP::RAB-3 in all cholinergic neurons (Fig. 1C), we again saw the large lateral synapses that VC4 and VC5 make onto the vm 2 cells. We also observed additional cholinergic presynaptic termini close to the vm 2 muscle arms that extend along the right ventral cord. Most of these are likely to be $\mathrm{VC}$ synapses onto the vm2 cells, but a few may represent the synapses of the VA7, VB6, and VD7 ventral cord motor neurons onto the vm1 muscles (White et al., 1986).

\section{The ERG K ${ }^{+}$channel inhibits $C$. elegans egg-laying muscle activity}

We screened for $C$. elegans mutants with increased egg-laying behavior, which could result from increased presynaptic excitation or from increased postsynaptic excitability of the vulval mus-
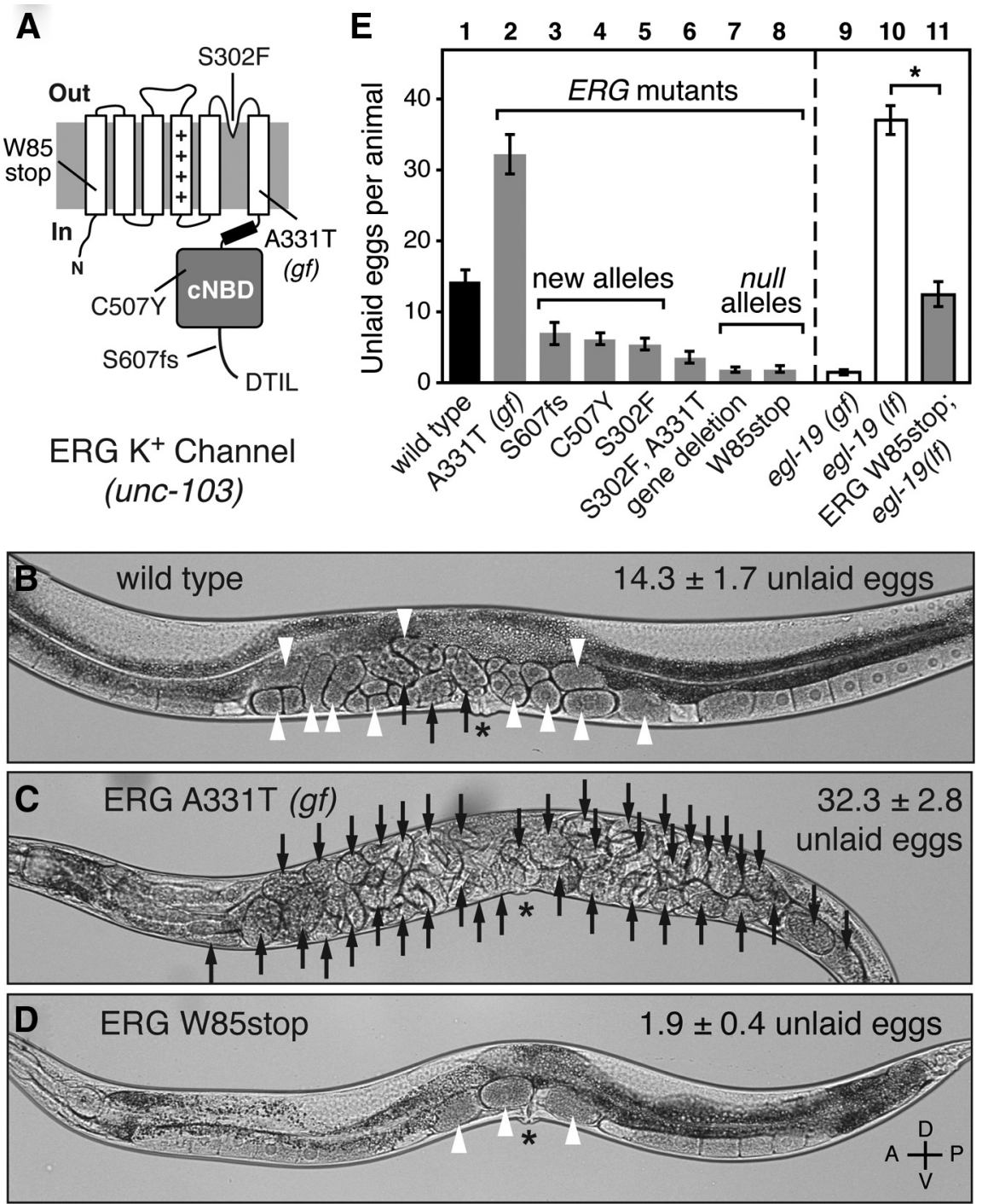

Figure 2. The ERG $\mathrm{K}^{+}$channel inhibits C. elegans egg-laying behavior. $\boldsymbol{A}$, Diagram of the C. elegans ERG $\mathrm{K}^{+}$channel and location of mutations. ++++ , voltage-sensor; fs, predicted frameshift mutation; gf, gain-of-function mutation; DTIL, predicted carboxy-terminal PDZ-interaction motif. The W85stop mutant is used as the standard ERG-null background in this work. $\boldsymbol{B}-\boldsymbol{D}$, Brightfield micrographs of wild-type C. elegans $(\boldsymbol{B})$, the A331T ERG gain-of-function mutant $(\boldsymbol{C})$, or the W85stop ERG-null mutant $(\boldsymbol{D})$. Black arrows indicate eggs with $>8$ cells, and white arrowheads indicate early-stage eggs of $\leq 8$ cells. The average number of unlaid eggs per animal $(n=30)$ is indicated $\pm 95 \%$ confidence intervals. The A331T gain-of-function mutant accusince it lays eggs too frequently. Asterisk, vulva; axes indicating anterior (A), posterior (P), dorsal (D), and ventral (V) are shown. Quantification of unlaid eggs in animals bearing ERG K ${ }^{+}$channel and L-type $\mathrm{Ca}^{2+}$ channel mutations. Wild-type animals (bar unction ( $g f$ ) and loss-of-function (If) mutations in egl-19, which encodes the sole $\alpha_{1}$ subunit of the C. elegans L-type $\mathrm{Ca}^{2+}$ channel, were tested individually or in combination with an ERG-null mutation. Error bars, $95 \%$ confidence intervals. Asterisk, $p<$ 0.0001 ( $t$ test $) ; n=30$ animals per genotype.

cles. In our first screen, we isolated mutants that lay their eggs prematurely (Bany et al., 2003). The embryos from these mutants are laid shortly after fertilization, when they are still at early stages of development. We separately screened for mutations that suppress a block in neurotransmitter release from HSN caused by an egl-47(dm) mutation (Tanis et al., 2009). One mutation from the first screen and two mutations from the second screen all mapped to the location of the $u n c-103$ gene and failed to complement each other as well as an $u n c-103$-null mutation. Sequencing revealed that each mutant had a single base-pair lesion in the unc-103 gene.

unc-103 is the sole C. elegans ortholog of ERG, which encodes a conserved, voltage-gated $\mathrm{K}^{+}$channel that inhibits cell excitabil- 
A

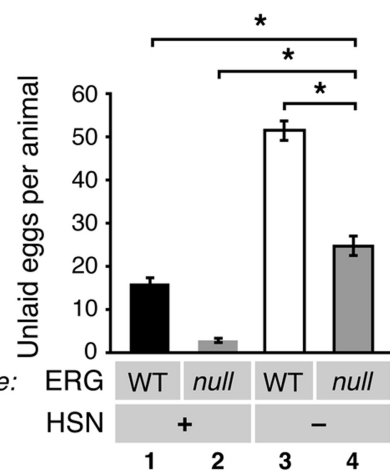

C

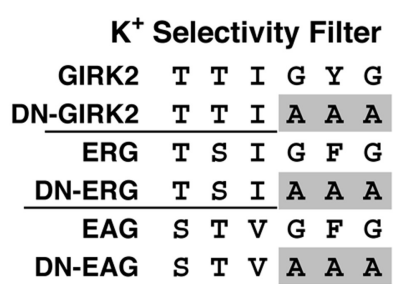

B

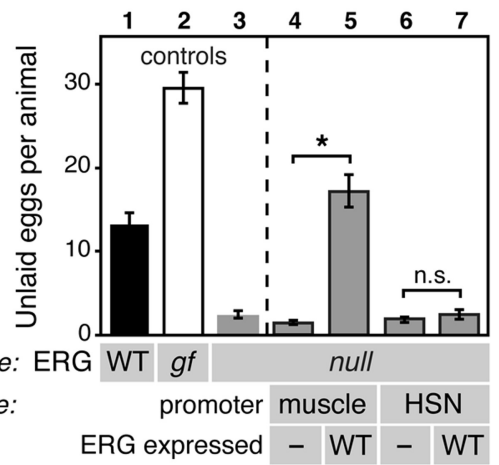

viously known unc-103-null mutants in showing well coordinated locomotion but increased egg laying, as evidenced by retaining fewer unlaid eggs (Fig. 2D,E), and laying eggs at early stages of development (data not shown). These and other previously reported results (Reiner et al., 2006) show that the ERG $\mathrm{K}^{+}$channel normally inhibits C. elegans egg-laying behavior.

C. elegans ERG may act to oppose membrane depolarization mediated by L-type $\mathrm{Ca}^{2+}$ channels. In the human heart, hERG acts to repolarize the heart muscle during long ventricular action potentials mediated by L-type $\mathrm{Ca}^{2+}$ channels (Sanguinetti and Tristani-Firouzi, 2006). In C. elegans, $\mathrm{L}-$ type $\mathrm{Ca}^{2+}$ channels mediate action potentials and are encoded by the egl-19 gene (Gao and Zhen, 2011; Liu et al., 2011). Gain-of-function egl$19(g f)$ mutations increase $\mathrm{Ca}^{2+}$ channel activity, causing excessive muscle excitability and hyperactive egg laying (Lee et al., 1997). The egl-19(gf) hyperactive egglaying phenotype was similar to that of ERG-null mutants (Fig. 2E, compare bars 8 and 9). Conversely, an egl-19(lf) mutant with decreased $\mathrm{Ca}^{2+}$ channel activity accumulated unlaid eggs, similar to animals bearing the A331T gain-of-function ERG mutation (Fig. 2E, compare bars 10 and 2 ). We found that combining loss-offunction mutations for ERG and EGL-19 that individually caused opposite egglaying defects led to an intermediate phenotype close to that of the wild type (Fig. $2 E$, bar 11). The reduction in unlaid eggs in the unc-103(W85stop), egl-19(lf) double mutant was not caused by a defect in egg production, as average brood sizes in the single and double mutants were not statistically different (egl-19(lf), $197 \pm 12$; unc-103 (W85stop), $196 \pm$ 30; unc-103

ity (Garcia and Sternberg, 2003; Reiner et al., 2006). As shown in Figure 2A, ERG channels have six transmembrane domains, including a voltage sensor, and a cytoplasmic cyclic nucleotide binding domain (cNBD). C. elegans $\mathrm{ERG}$ is $\sim 70 \%$ identical to the human ERG (hERG) in the transmembrane segments and cNBD. Although C. elegans ERG lacks the N-terminal PAS [period (PER)/Aryl hydrocarbon receptor nuclear translocator (ARNT)/ single-minded (SIM)] domain found in other ERG and EAG channels, it bears a PDZ [postsynaptic density-95 (PSD-95)/ Discs large (Dlg)/zona occludens-1 (ZO-1)]-interaction motif at its $\mathrm{C}$ terminus that may bind other proteins to mediate subcellular localization. unc-103 was originally identified by a gain-offunction mutation in the sixth transmembrane domain (A331T), which causes uncoordinated locomotion and a block in egg laying (Fig. 2C). hERG channels bearing this mutation (A653T) open more readily upon membrane depolarization (Petersen et al., 2004). Thus, the A331T gain-of-function mutation likely increases ERG K ${ }^{+}$channel activity and thereby inhibits the electrical excitability of cells that express it. Our three newly identified ERG mutations (Fig. $2 \mathrm{~A}$ ) were phenotypically similar to two pre-
(W85stop), egl-19(lf), $165.3 \pm 39)$. These results suggest that $C$. elegans ERG, like hERG in human heart muscle, antagonizes excitation caused by L-type $\mathrm{Ca}^{2+}$ channels.

We next determined in which cells ERG acts to inhibit egglaying behavior. The HSN motor neurons release serotonin and promote the induction of the active phase of egg-laying behavior. Animals lacking the HSNs are egg-laying defective, accumulating 50 unlaid eggs (Fig. 3A). This strong egg-laying defect of HSN (-) animals was partially suppressed in ERG-null mutants, but the animals remained egg-laying defective, accumulating $\sim 25$ eggs, significantly more than the wild type (Fig. $3 A$ ). This result shows that ERG acts at least in part outside the HSN to promote egg laying, but that ERG-null mutants also require HSN function to show hyperactive egg-laying.

Previous work has shown that six distinct promoters $(a-f)$ drive expression of ERG isoforms with different $\mathrm{N}$ termini in all muscle cells and many neurons, including the vulval muscles and HSN (Garcia and Sternberg, 2003; Reiner et al., 2006). We found that re-expression of the vulval muscle-expressed "e" isoform of ERG in vulval muscle using the muscle-specific myo-3 promoter 
fully rescued the hyperactive egg-laying defect of ERG-null mutants (Fig. 3B, compare bars 4 and 5). Re-expression of the HSN-expressed " $\mathrm{f}$ " isoform of ERG in HSN using the tph-1 promoter was unable to rescue (Fig. 3B, bars 6 and 7). These results indicate that ERG function in muscle is sufficient to inhibit egg-laying behavior.

To test where ERG function was necessary, we analyzed egg-laying behavior in animals transgenically expressing a dominant-negative ERG mutant. We adapted a previously described dominantnegative mutant of the GIRK2 $\mathrm{K}^{+}$channel (Kuzhikandathil and Oxford, 2000) in which mutation of the GYG residues in the $\mathrm{K}^{+}$selectivity filter to three alanines (AAA) dominantly blocks activity of wildtype GIRK2 subunits that assemble into tetramers with the mutant (Fig. 3C, left). We mutated the corresponding GFG in ERG to AAA and expressed this DN-ERG in wild-type animals from either the muscle-specific or HSN-specific promoters. Expression of DN-ERG in muscle caused hyperactive egg-laying (Fig. 3C, compare bars 4 and 5). This effect was specific to ERG, as expression of the analogous dominant-negative mutant of the related EAG channel EGL-2 had no effect (Fig. 3C, bar 6), even though EGL-2 is also expressed in the vulval muscles (Weinshenker et al., 1999). Expression of DN-ERG in the HSN caused a significant reduction in the number of unlaid eggs, suggesting that ERG does regulate the electrical excitability of the HSN. However, this phenotype was weaker than that seen for muscle-specific inactivation of ERG.

Together, our results show that ERG acts in vulval muscles to inhibit egg-laying behavior, presumably by inhibiting the electrical excitability of these muscles. ERG has an additional, weaker effect inhibiting egg laying by acting in the HSN neurons.

ERG localizes to the vulval muscle postsynaptic termini via a PDZ-interaction motif

We found that within vulval muscles, ERG is subcellularly enriched at postsynaptic termini. We transgenically expressed a fusion protein in which GFP was inserted into ERG just upstream of its C-terminal PDZinteraction motif. The unc-103e promoter (Reiner et al., 2006) was used in a single-copy, chromosomally inserted transgene to achieve low-level expression specifically in the vulval muscles. We used a control transgene to express a red fluorescent protein, consisting of the murine CD8 protein fused to mCherry, which is passively distributed within the plasma membrane of the vulval muscles. Ratiometric confocal fluorescence imaging showed that
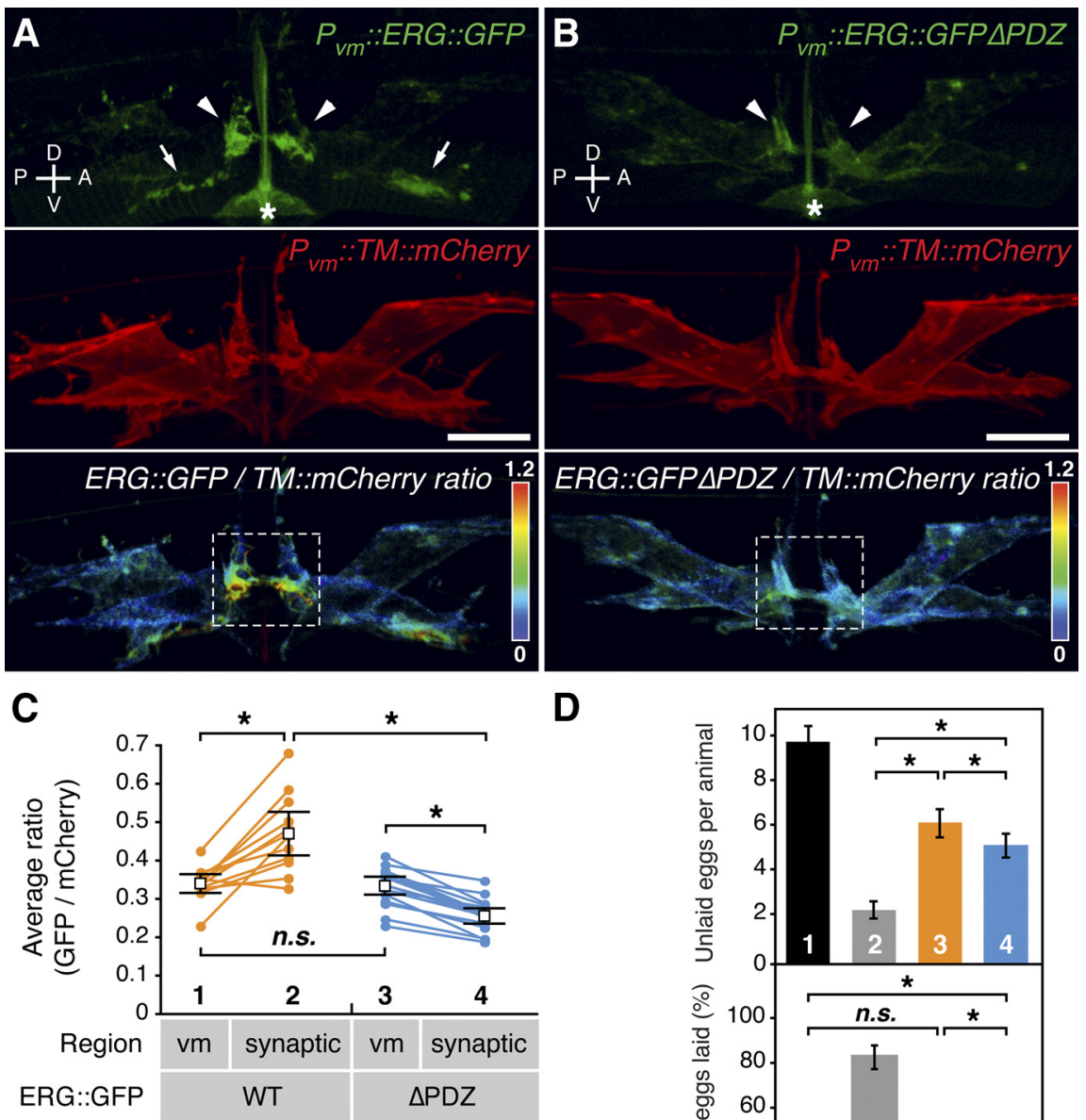

D

Figure 4. ERG is localized in vulval muscle to the postsynaptic termini via a PDZ-interaction motif. $A, B$, Representative ratiometric images of ERG localization. $A$, ERG bearing internally inserted GFP (ERG::GFP) was expressed in vulval muscle from the unc-103e promoter $\left(P_{\mathrm{vm}}\right)$. Its localization (top) was compared with that of a coexpressed transmembrane control protein (TM::mCherry, middle) using ratiometric imaging (bottom). $B$, An ERG mutant lacking the putative PDZ-interaction motif (-DTIL) at its carboxy terminus (ERG::GFP $\triangle$ PDZ) is shown as in $\boldsymbol{A}$. ERG::GFP was enriched at lateral postsynaptic sites (arrowheads) and along the ventral cord (arrows). Dashed white boxes indicate regions of lateral vm2 processes selected for quantitation in $\boldsymbol{C}$. Asterisks, the autofluorescent vulval slit; scale bar, $10 \mu \mathrm{m}$; rainbow scale, GFP/mCherry ratio. C, Statistical analysis of ratiometric imaging. ERG::GFP to TM::mCherry ratios for entire set of vulval muscles (vm) on one side of an animal are compared with the ratio within the lateral synaptic subregion ( $\boldsymbol{A}, \boldsymbol{B}$, white boxes). Paired measurements from the same animal are connected by lines; $n=$ 12 for wild-type ERG and $n=17$ for the $\triangle P D Z$ mutant. Average ratio pairs are shown; asterisks, $p<0.0004$ ( $t$ test); n.s., not significant with $p>0.05$. D, Postsynaptic enrichment of ERG is required for proper inhibition of egg laying. Wild type (bar 1$)$ or the W85stop ERG-null mutants (bars 2-4) bearing transgenes expressing either wild-type ERG::GFP (bar 3) or the ERG::GFP $\triangle P D Z$ mutant (bar 4 ) from the vm-specific promoter were assayed for egg-laying behavior. Top, Unlaid eggs per animal; asterisks, $p<$ 0.0001 ( $t$ test; $n=60$ animals per genotype). Bottom, Percentage of eggs laid at early stages ( $\leq 8$ cells); asterisks, $p<0.0001$; n.s., not significant with $p>0.05$ (Fisher's exact test; $n>195$ eggs per genotype). Error bars, $95 \%$ confidence intervals. Axes indicating anterior (A), posterior (P), dorsal (D), and ventral (V) are shown.

ERG::GFP was enriched at two locations within the vm2 that correspond to the two different postsynaptic sites in these muscles (Fig. $4 A$ ). First, we saw enrichment at the ends of the lateral processes that serve as the postsynaptic termini onto which the HSN and VC neurons make synaptic contacts. Second, we saw localization along the muscle arms of the right vm2s onto which the right ventral cord neurons make synapses. In addition to being enriched at the postsynaptic termini, ERG::GFP was also 


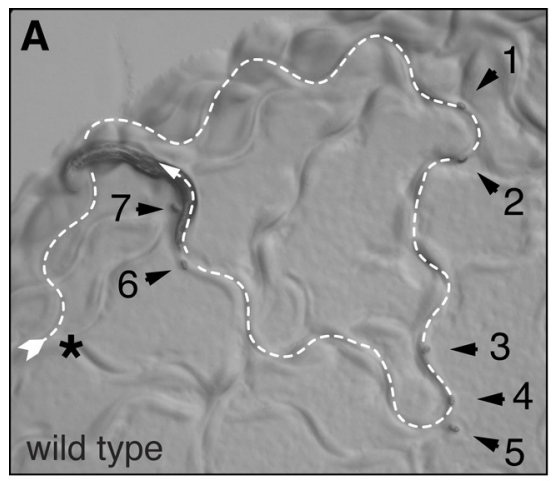

B
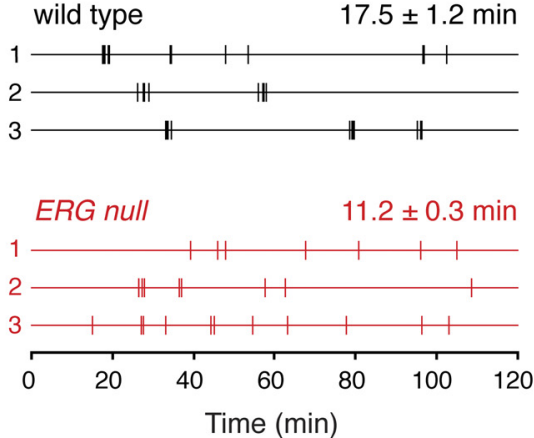

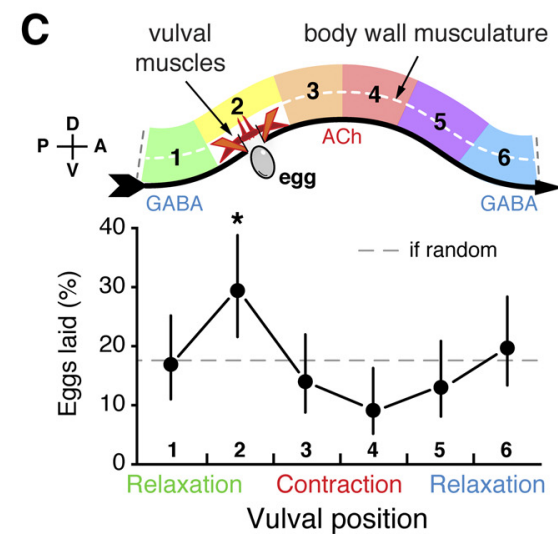

Figure 5. Egg laying occurs in active phases, is rhythmically phased with the body bends of locomotion, and occurs at shorter intervals in ERG-null mutants. $\boldsymbol{A}$, Eggs are laid during brief active phases. Micrograph of a wild-type worm on a standard NGM agar plate with a lawn of bacterial food during the active phase of egg laying. Worm anterior is to the left, the dashed arrow indicates the track of worm locomotion, and arrowheads show seven eggs laid during one active phase. $\boldsymbol{B}$, Egg laying on standard NGM agar plates with food was followed by video recording for $2 \mathrm{~h}$ in each of six wild-type and six ERG-null (W85stop) mutant animals. Videos were reviewed to identify the specific time points when each egg was laid (vertical hash marks), and representative data from three wild-type animals (black) and three ERG-null mutant animals (red) are shown (lines 1-3). The wild type typically lays multiple eggs during a $<4$ min active phase followed by a longer interval with no egg laying. ERG-null mutants typically lay only a single egg per active phase, likely because they contain only $\sim 2$ eggs in their uterus, of which only one may be in position to be laid. We determined the intercluster time constant from the slope of the log-tail distribution of intercluster intervals ( $>4$ min), as previously described (Waggoner et al., 1998). The deduced intervals are shown \pm SEM and are significantly different ( $p<0.0001$, analysis of covariance). $C$, Egg laying is phased with the sinusoidal body bends that drive locomotion. Diagram (top) of the vulval muscles and ventral body-wall muscles (dashed lines) during one sinusoidal wavelength of locomotion as the animal moves to its anterior on right (ventral arrow). This idealized wave was segmented into six regions (1-6). Bottom, From video recordings from each of six wild-type animals ( $2 \mathrm{~h}$ each), the vulval position during 104 egg-laying events was determined. The proportion of eggs laid at each body bend region is shown; asterisks, $p=0.0058$ (region 2), $p=0.039$ (region 4, Fisher's exact test). Error bars, $95 \%$ confidence intervals. Axes indicating anterior (A), posterior ( $P$ ), dorsal (D), and ventral (V) are shown.

found at lower levels uniformly over the vulval muscle plasma membrane. Quantifying the ERG::GFP to membrane mCherry ratio in voxels from a subregion containing the vm2 lateral processes of multiple animals showed that ERG::GFP is significantly enriched at the lateral postsynaptic region compared with its levels averaging voxels over the entire vulval muscle (Fig. $4 C$, compare 1 and 2).

To test whether the synaptic enrichment of ERG was mediated by its C-terminal PDZ-interaction motif, we generated another single-copy transgene that expressed an ERG::GFP $\Delta$ PDZ mutant in which the last four residues (DTIL) were deleted. This mutant protein lacked the synaptic enrichment seen for wild-type ERG::GFP (Fig. 4B). Indeed, ERG::GFP $\Delta$ PDZ was actually depleted from the postsynaptic terminus (Fig. 4C, compare 3 and 4). This was not due to a change in expression levels, as the ratio of GFP to mCherry over the entire vulval muscle was identical in the strains expressing wild-type ERG::GFP versus ERG::GFP $\triangle$ PDZ (Fig. 4C, compare 1 and 3). These results show that ERG localizes in vulval muscle to postsynaptic termini via a PDZ interaction motif, presumably by binding an unidentified PDZ domain-containing protein localized to synapses.

To test whether ERG localization to the synapse is important for its function, we compared the ability of wild-type and $\triangle \mathrm{PDZ}$ ERG::GFP single-copy transgenes to rescue the hyperactive egglaying defect of ERG-null mutants. Re-expression of wild-type ERG::GFP partially rescued the number of unlaid eggs toward wild-type levels, while the ERG::GFP $\triangle \mathrm{PDZ}$ mutant produced less rescue in this assay (Fig. $4 D$, top, compare bars 3 and 4 ). These data are compromised by the fact that ERG-null mutants fail to accumulate eggs both due to hyperactive egg-laying and due to a failure to produce a normal number of eggs; the latter defect cannot be rescued by re-expression of ERG just in the vulval muscles. Thus, in a second assay of egg-laying behavior less sensitive to defects in egg production, we measured the developmental stage of freshly laid eggs (Chase and Koelle, 2004). While wild-type animals laid about $10 \%$ of their eggs at early stages of development, ERG-null mutants laid $>80 \%$ of their eggs at early stages (Fig. 4D, bottom, compare bars 5 and 6). Re-expression of wild-type ERG::GFP in the vulval muscles completely rescued this measure of hyperactivity, while the ERG::GFP $\triangle P D Z$ mutant was unable to fully rescue. Previous work has identified an ERG isoform generated by alternative splicing that leads to the premature termination of the protein upstream of the PDZ-interaction motif (Reiner et al., 2006). This suggests that some native ERG channels may be functional outside of PDZ complexes, and indeed we see significant rescue when ERG::GFP $\triangle \mathrm{PDZ}$ mutant is the sole ERG expressed in the vulval muscles (Fig. 4D). However, our results show that the ERG " $e$ " isoform expressed in vulval muscle is enriched at the postsynaptic terminus via its PDZinteraction motif and that this localization is required for proper inhibition of egg-laying behavior.

\section{Vulval muscle contractions are phased with respect to body bends and inhibited by ERG}

To understand more precisely how ERG affects egg-laying behavior, we video recorded and quantitatively analyzed this behavior in wild-type and ERG-null mutant animals as they moved freely on the standard NGM agar plates used for C. elegans growth in the laboratory. As seen previously (Waggoner et al., 1998), wild-type animals enter an active phase for several minutes, during which a cluster of eggs is laid, followed by an intercluster interval with no egg laying that lasts about 20 min until the start of the next active phase (Fig. 5A,B). ERG-null mutants usually laid single eggs rather than clusters of eggs, probably in part because the adults have only $\sim 2$ eggs in their uterus, of which only one may be in position to be laid (Fig. $5 B$ ). However, $\sim 25 \%$ of egg-laying events in the ERG-null mutant were in clusters of $\geq 2$ eggs (Fig. $5 B$, data not shown), indicating the ERG-null mutant does have active phases for egg laying. Loss of ERG reduced the interval between egg-laying events. In wild-type animals, this interval averaged $17.5 \pm 1.2 \mathrm{~min}$, while in ERG-null mutants it was reduced to $11.2 \pm 0.3 \mathrm{~min}$ (Fig. $5 B$ ). This direct observation of the increased 

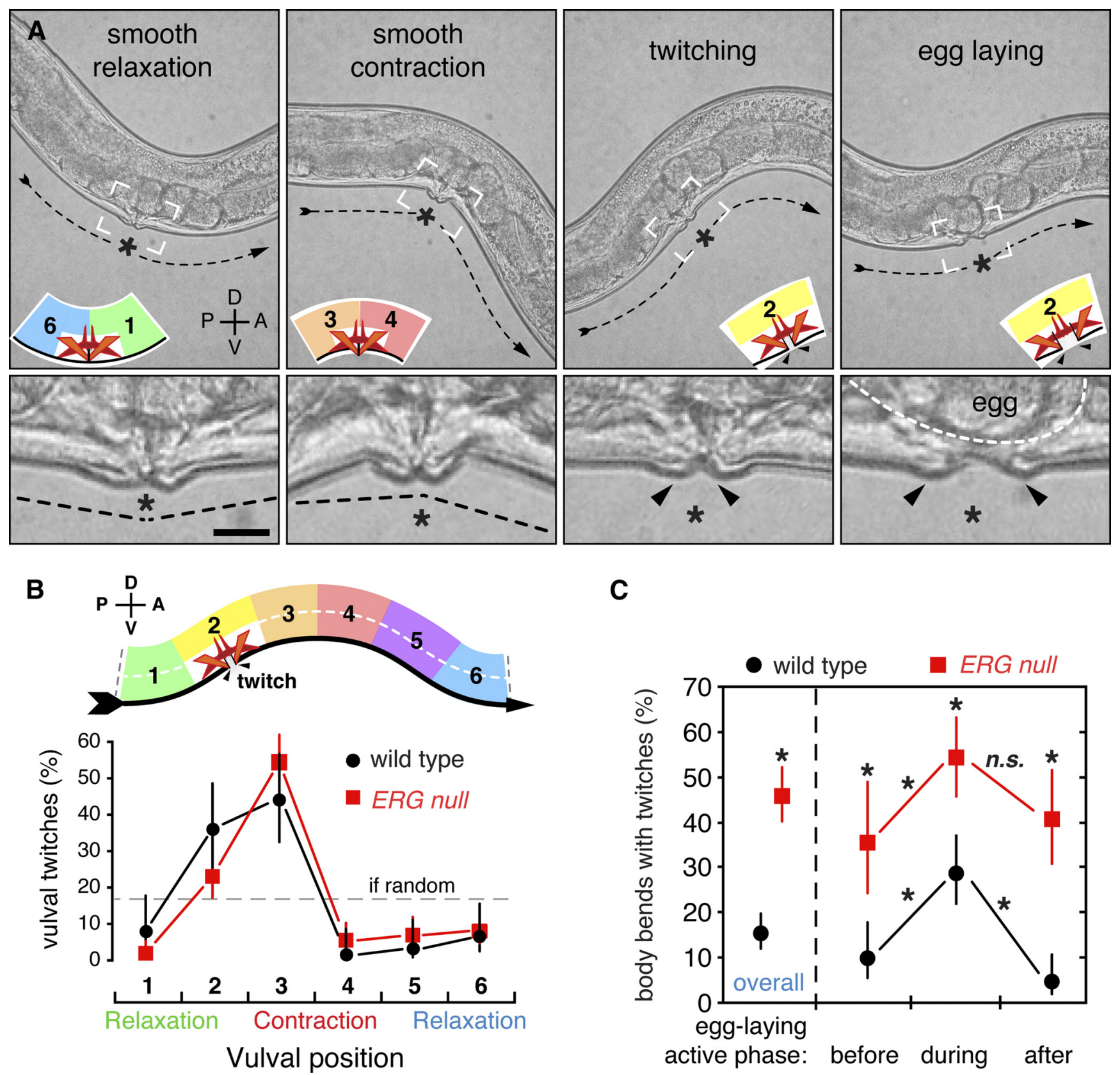

C

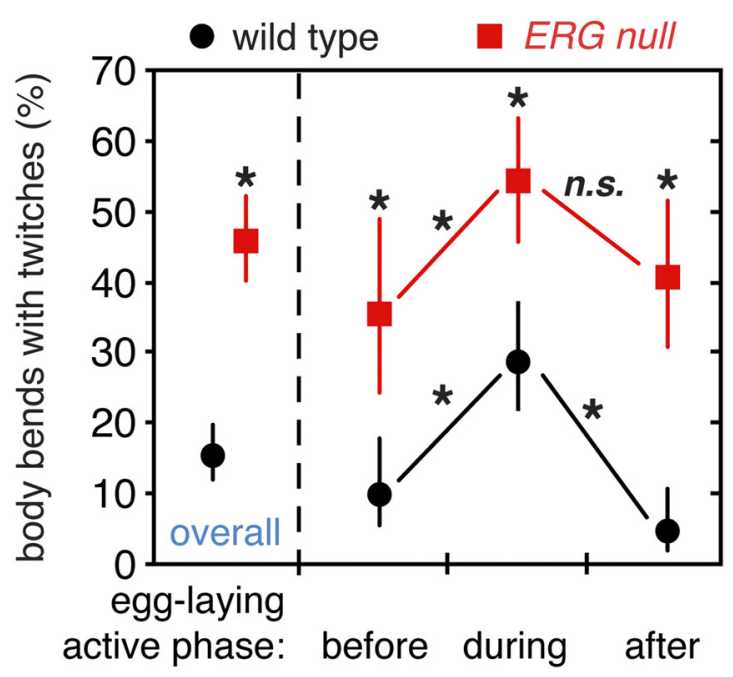

Figure 6. Vulval muscle contractions are phased with respect to locomotor body bends and are limited by ERG. $A$, High-speed video recordings of freely behaving worms reveal four vulval muscle excitability states: smooth relaxation, smooth contraction, twitching, and egg laying. Top, Still frames are shown from an ERG-null mutant recording where the twitches are more easily seen compared with those of wild-type animals (Movie 1). Dashed arrows indicate worm direction and posture. Smooth relaxation occurs when the vulva passes through the ventral relaxation trough while smooth contraction occurs at the ventral contraction crest. Twitches are visible vulval muscle contractions that move and separate the tips of the vulva, while egg laying accompanies full vulval opening and egg release. Inset diagrams indicate position of the vulva within the idealized wave, and white boxes indicate regions of high-magnification insets (bottom). Asterisk, vulva; arrowheads, opening of the vulva during twitching and egg laying. Scale bar, $10 \mu \mathrm{m}$. B, Vulval muscle twitching is phased with locomotion. High-speed video was used to record the timing of twitches in five wild-type and five ERG-null mutant animals. Each video included $5 \mathrm{~min}$ before and $7 \mathrm{~min}$ after the first egg-laying event. Twitches were mapped relative to the timing of vulval muscle smooth contractions and relaxations (top) as it moves to its anterior on right (ventral arrow). Points indicate the proportion of twitches that occurred at each position (wild type: black circles, $n=$ 61 twitches; ERG-null mutants: red squares, $n=160$ twitches). C, Vulval muscle twitching increases during egg laying and is more frequent in ERG-null mutants. From the high-speed data in $\boldsymbol{B}$, the frequency of body bends with twitches was determined. One minute before and 3 min after the first egg-laying event was designated as the active phase for paired analyses; asterisks, $p<0.0001$ (Fisher's exact test); n.s., not significant ( $p>0.05)$. Error bars, 95\% confidence intervals. Axes indicating anterior (A), posterior (P), dorsal (D), and ventral (V) are shown.

egg laying by the ERG-null mutant accounts for the phenotypes observed in less direct assays of egg-laying behavior (Fig. 2E).

We noticed in our videos that eggs were often laid during locomotion when the vulva was at a particular phase of a body bend. C. elegans moves with sinusoidal body bends consisting of waves of body-wall muscle contraction and relaxation driven by ventral nerve cord motor neurons. ACh drives contraction while
GABA causes relaxation (Fig. 5C, top). We subdivided an idealized waveform into six parts and determined which subdivision contained the vulva at the time each egg was laid. We found that egg laying increased in frequency as the vulva neared a ventral contraction and was subsequently inhibited until after the vulva passed through this contraction (Fig. $5 C$ ). We saw a similar trend for ERG-null mutants (data not shown). 


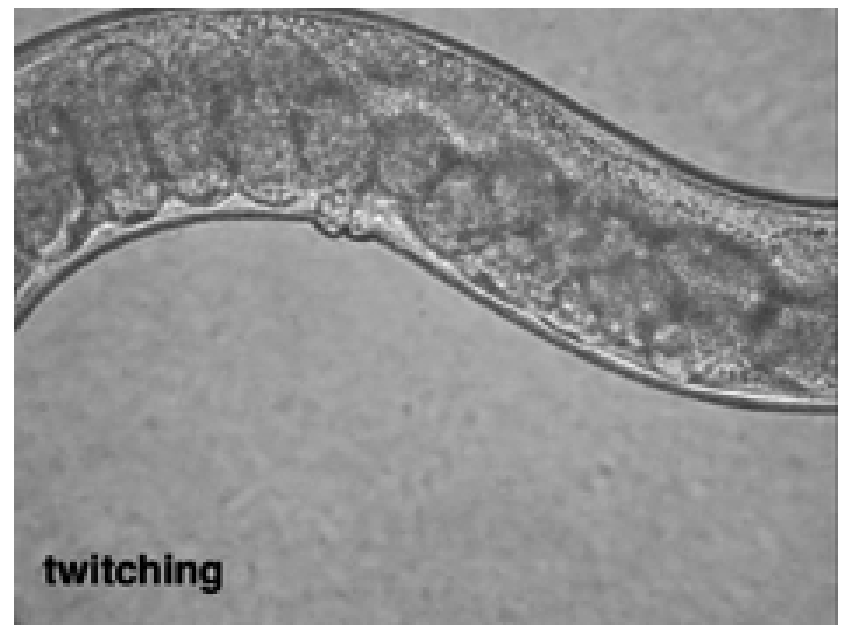

Movie 1. Rhythmic vulval muscle twitching contractions in wild-type animals during the egg-laying active phase. Bright-field, high-speed video of behaving wild-type C. elegans during locomotion, twitching, and egg-laying behavior. Video was recorded at $120 \mathrm{fps}$, and is shown at $30 \mathrm{fps}$ (4-fold slower than real time). See also Figure 6.

For a higher-resolution analysis, we developed conditions to directly observe vulval muscle contractions in behaving animals. The experiments shown in Figure 5, like almost all previous studies of C. elegans egg-laying behavior, infer the occurrence of vulval muscle contractions indirectly and only when they result in an egg being laid. Using a relatively high-magnification $(40 \times)$ objective lens, video recording at $120 \mathrm{fps}$ to capture rapid contractions, and continuously moving the microscope stage to keep the vulva in the field of view, we could directly view vulval muscle contractions as worms moved. We found that in both the wildtype and in ERG-null mutants, the vulval muscles undergo three distinct types of contraction (Fig. $6 \mathrm{~A}$ and Movie 1). First, we observed slow, smooth contractions and relaxations during every body bend similar to those of the adjacent body-wall muscles. The slow, smooth vulval movements might be due to active contraction of the vulval muscles themselves, or might simply result from passive compression and stretching of the vulval region as the adjacent body-wall muscles contract and relax during body bends. Second, we observed more rapid contractions, which we refer to as twitches, that visibly moved the vulva, and that often occurred independently on either the anterior or posterior side of the vulva (Fig. $6 \mathrm{~A}$ ). Finally, a third type of contraction, stronger than a twitch and always occurring on both anterior and posterior sides of the vulva, was used to release eggs. In the wild type, smooth contraction at the vulva occurred during every body bend (Movie 1), but as detailed below, twitches and egg-laying contractions occurred about 15 and $5 \%$ as frequently, respectively.

We found that twitches, like egg-laying events, were phased with respect to body bends. Because the narrow field of view of our high-magnification videos did not allow us to see an entire body-bend waveform, we determined the timing of the twitch relative to the smooth contractions and relaxations at the vulva (Fig. $6 B$ ). We found that twitching dramatically increased in frequency as the vulva neared a smooth contraction, and that twitching was subsequently inhibited until after a smooth relaxation (Fig. $6 \mathrm{~B}$ ). This phasing of twitching with locomotion was reminiscent of but stronger than the phasing observed for egg laying, and was unchanged in ERG-null mutants.

Strikingly, ERG-null mutants had three times as many vulval twitches as did the wild type, with nearly half of all smooth con- tractions in the ERG-null mutant preceded by a twitch compared with only $15 \%$ in the wild type. (Fig. 6C). We further analyzed whether twitch frequency changed during the active periods of egg laying. In wild-type animals, vulval twitches became 2-3-fold more frequent during the active phase of egg laying, defined as a $4 \mathrm{~min}$ period surrounding an egg-laying event (Fig. $6 \mathrm{C}$ ), and then declined back to a low level. In ERG-null mutants, vulval twitching also increased during active periods of egg laying and then declined afterward.

Together, these results suggest the hypothesis that the vulval muscles, like adjacent body-wall muscles, receive an excitatory signal at a particular phase of every body bend. This rhythmic excitation sometimes results in a twitch or, more rarely, an egglaying contraction, and the probability of a twitching or egglaying response becomes higher during the active phase of egg laying. Our results show that ERG acts both during and between active phases to lower the probability that the rhythmic excitation leads to twitching or egg-laying responses.

\section{Distinct, rhythmic $\mathrm{Ca}^{2+}$ transients in vulval muscles underlie twitches and egg-laying contractions}

To more directly examine excitation of vulval muscles during locomotion, twitching, and egg laying, we coexpressed the $\mathrm{Ca}^{2+}$ sensitive green fluorescent protein GCaMP3 (Tian et al., 2009) and the $\mathrm{Ca}^{2+}$-insensitive red fluorescent protein mCherry in the vulval muscles and performed confocal imaging of behaving animals (Fig. 7A-E, Movies 2, 3). We found we could track the smooth contractions and relaxations of the vulval muscles during locomotion as changes in the area of mCherry fluorescence (Fig. $7 A, B, F)$. By calculating the ratio of GCaMP3/mCherry fluorescence, we could detect $\mathrm{Ca}^{2+}$ transients while correcting for changes in its fluorescence intensity caused by changes vulval muscle size, variations in focus, and movement (Fig. $7 F$ ).

During the egg-laying active phase, we detected large $\mathrm{Ca}^{2+}$ transients in the vulval muscles accompanying most body bends (Fig. 7F, right), as predicted from our analysis of egg-laying muscle contractions. Such fluorescence ratio changes were not observed in animals expressing the $\mathrm{Ca}^{2+}$-insensitive GFP, even when they executed strong egg-laying contractions (Fig. $7 G$ ), indicating that our recordings detect genuine $\mathrm{Ca}^{2+}$ transients and that effects of movement were removed by the ratiometric analysis used. During the egg-laying inactive phase, body bends typically lacked $\mathrm{Ca}^{2+}$ transients (Fig. 7F, left), further demonstrating that the GCaMP3/mCherry ratio transients we observed were not simply a consequence of animal movement or changes in vulval muscle size.

Just as egg-laying events (Fig. 5C) and vulval muscle twitches (Fig. $6 B$ ) are phased with respect to locomotor body bends, vulval muscle $\mathrm{Ca}^{2+}$ transients also showed similar phasing (Fig. $7 \mathrm{H}$ ), arguing that all result from the same rhythmic excitation of the egg-laying muscles that appears to occur at a particular phase of each body bend.

We plotted the peak amplitudes of all the $\mathrm{Ca}^{2+}$ transients in our recordings (Fig. 7I) and found that they clustered into two distinct groups, which we named twitch and egg-laying transients. In wild-type animals, the average change in GCaMP3/ mCherry ratio $(\Delta R / R)$ was $\sim 50 \%$ for twitch transients, and $200 \%$ for egg-laying transients.

Twitch $\mathrm{Ca}^{2+}$ transients (Fig. 7C,D) appeared to underlie the twitching contractions observed during our bright-field movies of vulval muscle contractions. Like twitching contractions, twitch $\mathrm{Ca}^{2+}$ transients sometimes occurred on only the anterior or posterior side of the vulva (Fig. 7C; Movies 2, 3). Twitch $\mathrm{Ca}^{2+}$ tran- 

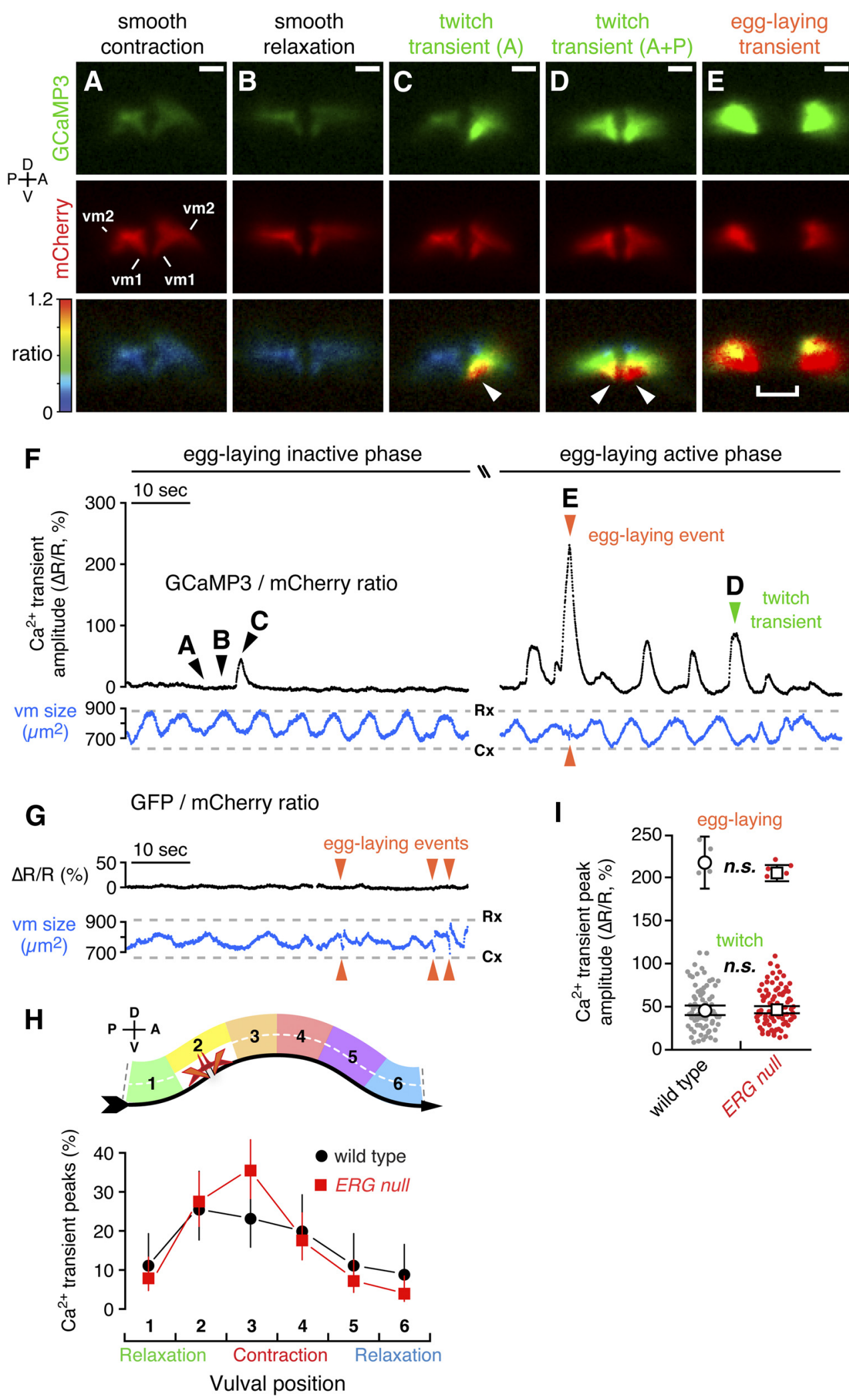

Figure 7. Distinct vulval muscle $\mathrm{Ca}^{2+}$ transients mediate twitching and egg-laying behaviors. $\boldsymbol{A}-\boldsymbol{E}$, Ratiometric $\mathrm{Ca}^{2+}$ imaging in the vulval muscles in behaving animals. Three 1 min recordings were made for each of 10 wild-type and 10 ERG-null animals. GCaMP3 (top) and mCherry (middle) fluorescence, and the GCaMP3/mCherry intensity-modulated ratio (bottom) images from a wild-type animal are shown and correspond to the time points indicated by arrowheads labeled A-E in $\boldsymbol{F}$. C, A twitch transient in the anterior half of the vulva. $\boldsymbol{D}$, A twitch transient occurring in both anterior and posterior halves. Arrowheads, Subcellular localization of the GCaMP3/mCherry ratio peak at the vulval tips. Separation of the two halves of the vulva in $\boldsymbol{E}$ is due to contraction of the vulval muscles and the passage of an egg through the vulva. Scale bar, $10 \mu \mathrm{m}$; positions of vm1 and vm 2 are indicated. See also Movies 2 and 3 . F, Traces from a representative wild-type animal are shown of the average GCaMP3/mCherry ratio in the vulval muscles ( $\Delta R / R$, black trace) before (left side; Movie 2 ) and during the egg-laying active phase (right side; Movie 3). Traces of the area of the $\mathrm{mCherry} \mathrm{signal} \mathrm{(blue)} \mathrm{indicate} \mathrm{changes} \mathrm{in} \mathrm{vulval} \mathrm{muscle} \mathrm{(vm)} \mathrm{size} \mathrm{during} \mathrm{locomotion.} \mathrm{Cx,} \mathrm{smooth} \mathrm{contraction;} \mathrm{Rx,} \mathrm{smooth} \mathrm{relaxation.} \mathrm{Arrowheads} \mathrm{indicate} \mathrm{Ca}{ }^{2+}$ transients that mediate egg-laying and twitching contractions. G, Ratiometric imaging in the vulval muscles in behaving animals expressing the $\mathrm{Ca}^{2+}$-insensitive GFP along with mCherry. Egg-laying (Figure legend continues.) 


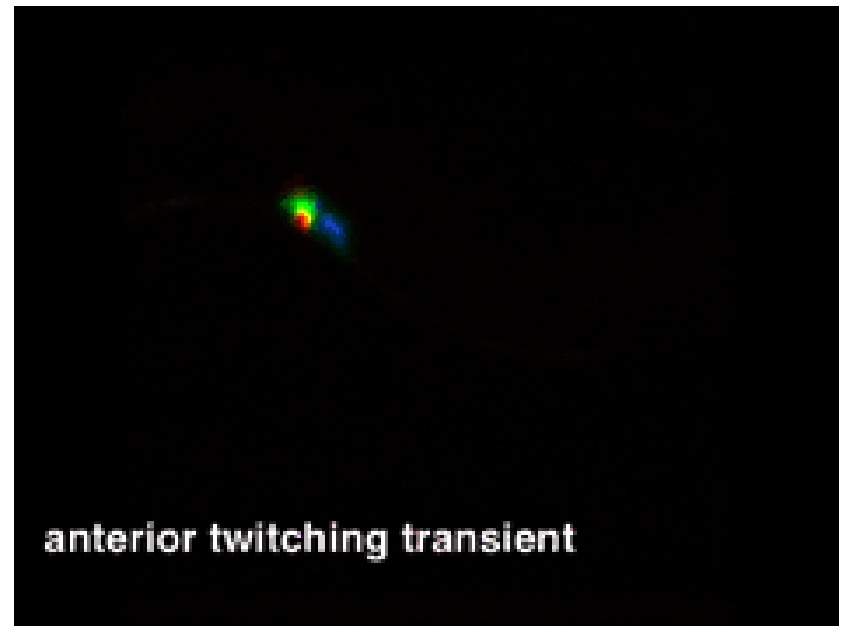

Movie 2. Infrequent $\mathrm{Ca}^{2+}$ transients in the vulval muscles of wild-type animals before the egg-laying active phase. Intensity-modulated ratiometric imaging of wild-type C. elegans expressing the $\mathrm{Ca}^{2+}$ sensor GCaMP3 and soluble mCherry in the vulval muscles from the unc-103e promoter at $30 \mathrm{fps}$. Change in the GCaMP3 to mCherry fluorescence ratio is indicated by a rainbow scale from 0 (dark blue) to 1.2 (red). A twitch $\mathrm{Ca}^{2+}$ transient in the anterior vulval muscle cells was observed at $\sim 20$ s. Still images from this video are shown in Figure $7 A-C$, and the traces of $\Delta R / R$ and vulval muscle area are shown in Figure $7 F$ (left).

sients also increased in frequency during egg-laying active phases (Fig. 7F). Furthermore, twitching contractions were sometimes visible in the fluorescence recordings during twitch transients (Movie 3), despite the fact that the imaging conditions used were not well-suited to capturing such movements. Egg-laying $\mathrm{Ca}^{2+}$ transients (Fig. 7E, Movie 3) occurred simultaneously in both anterior and posterior vulval muscles, and were accompanied by the complete vulval openings that occur only during egg laying.

Twitch transients showed their peak magnitude at the ventral tips of the vulva (Fig. 7C,D, arrowheads; Movies 2, 3), a location distinct from the synaptic sites of the vulval muscles (Fig. 1). Egg-laying transients also initiated at the ventral tips of the vulva but then spread throughout the vulval muscles (Movie 3). These results suggest that the twitch and egg-laying $\mathrm{Ca}^{2+}$ transients observed do not mainly result from local $\mathrm{Ca}^{2+}$ influx at synapses, but instead result from the propagation of excitation to distal sites in the vulval muscles. We did not detect any statistically significant difference in the amplitude of vulval muscle $\mathrm{Ca}^{2+}$ transients upon loss of ERG (Fig. 7I). Thus, while ERG channels appear to determine how sensitively the vulval muscle respond to the rhythmic excitation that occurs during each body bend, once release of $\mathrm{Ca}^{2+}$ is triggered, ERG has no further effect. The synaptic enrichment of ERG (Fig. 4) is consistent with the idea that

\section{$\leftarrow$}

(Figure legend continued.) contractions (arrowheads) appear as events in the vulval muscle size trace (blue) but are silent in the GFP/mCherry ratio. $\boldsymbol{H}$, Vulval muscle $\mathrm{Ca}^{2+}$ transients are phased with locomotion. Six minute recordings were made for each of six wild-type and ERGnull animals. The peak of each $\mathrm{Ca}^{2+}$ transient was mapped relative to the timing of vulval muscle smooth contractions and relaxations (top) as the animal moves to its anterior on right (ventral arrow). Points indicate the proportion of transients that occurred at each position (wild type: black circles, $n=90$ transients; ERG-null mutants: red squares, $n=152$ transients). $I$, Peak GCaMP3/mCherry ratio $(\Delta R / R)$ amplitudes for $\mathrm{Ca}^{2+}$ transients recorded from three $1 \mathrm{~min}$ recordings from each of 10 wild-type and 10 ERG-null animals are shown in a scatter plot (gray, $n=85$ wild-type transients; red, $n=102$ ERG-null mutant transients). Transients were grouped by peak $\Delta R / R$ amplitude into egg-laying ( $>120 \%)$ and twitch $(0-120 \%)$ transients. Open circles (wild type) and squares (ERG null) indicate the mean amplitude for each group; $n$.s., not significant ( $p>0.05 ; t$ test). Error bars, $95 \%$ confidence intervals. Axes indicating anterior $(A)$, posterior $(P)$, dorsal $(D)$, and ventral $(V)$ are shown.

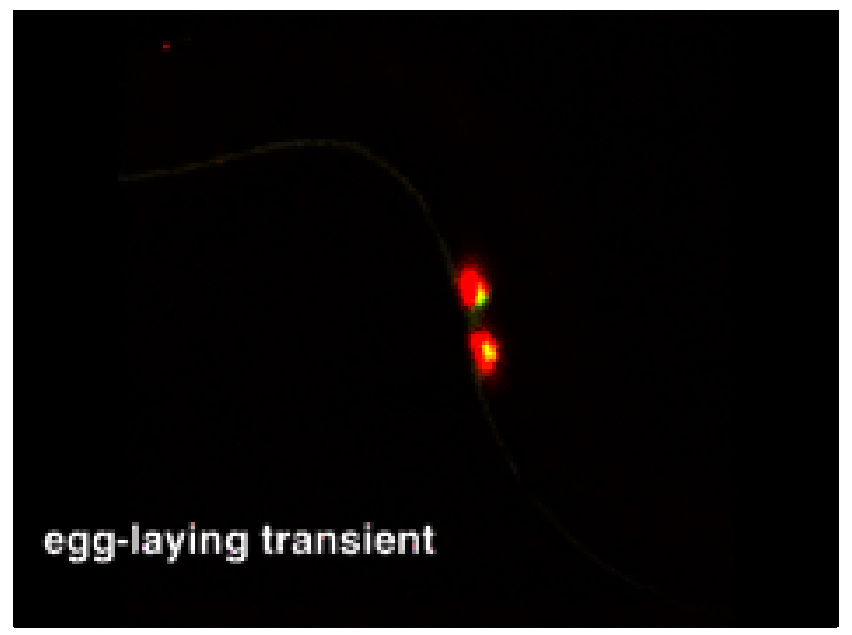

Movie 3. Frequent, rhythmic $\mathrm{Ca}^{2+}$ transients in the vulval muscles of wild-type animals during the egg-laying active phase. This is the same animal as shown in Movie 2 and Figure $7 A-F$, recorded approximately 4 min later. Change in the GCaMP3 to mCherry fluorescence ratio is indicated by a rainbow scale from 0 (dark blue) to 1.2 (red). Twitch and egg-laying $\mathrm{Ca}^{2+}$ transients are indicated. Still images from this video are shown in Figure $7 D, E$, and the traces of $\Delta R / R$ and vulval muscle area are shown in Figure $7 F$ (right). This video includes one egg-laying event at $\sim 15 \mathrm{~s}$

ERG specifically affects the initial excitation of the muscles at the egg-laying synapse.

\section{ERG mutants have more frequent vulval muscle $\mathrm{Ca}^{2+}$ transients}

To more fully capture changes in $\mathrm{Ca}^{2+}$ transient frequency during inactive and active egg-laying periods, we captured longer (6 min) recordings by adjusting our recording conditions to a slightly reduced temporal and spatial resolution. Twitch transients were sparse during the egg-laying inactive phase in wild-type animals (Fig. 8A), precisely as we found through our bright-field recordings of vulval muscle twitching (Fig. $6 \mathrm{C}$ ). $\mathrm{Ca}^{2+}$ transients became much more frequent as animals entered an egg-laying active phase and then decayed back to a low level (Fig. 8B). ERG-null mutants also showed active phases with an increased frequency of $\mathrm{Ca}^{2+}$ transients (Fig. 8D). However, ERG-null mutants had more frequent $\mathrm{Ca}^{2+}$ transients even during the inactive phase (Fig. $8 \mathrm{C}$ ). We analyzed the duration of intertransient intervals. Wild-type animals spent $\sim 51 \%$ of our recordings in inactive periods with $\leq 1$ $\mathrm{Ca}^{2+}$ transient per minute, while in ERG-null mutants, this inactive period was reduced to $\sim 36 \%$ of time spent (Fig. $8 E$ ). Conversely, wild-type animals spent $25 \%$ of our recordings in more active periods with $\mathrm{Ca}^{2+}$ transients occurring every $20 \mathrm{~s}$ or less, while in ERGnull mutants these active periods increased to $32 \%$ of time spent (Fig. 8 E). This increase in $\mathrm{Ca}^{2+}$ transient frequency in the ERG-null mutant is consistent with our behavioral results showing that ERGnull animals have more frequent egg-laying active phases and more frequent twitching contractions.

Together, our data indicate that in ERG-null mutants, the primary defect that leads to hyperactive egg-laying behavior appears to be increased postsynaptic excitability, such that rhythmic synaptic stimulation of vulval muscles during each body bend produces responses closer to the threshold required to trigger $\mathrm{Ca}^{2+}$ transients. This leads to more frequent vulval muscle twitches and more frequent egg-laying contractions.

\section{Discussion}

We integrated molecular genetics, assays of subcellular localization, and both bright-field and fluorescent $\mathrm{Ca}^{2+}$-indicator imag- 
A

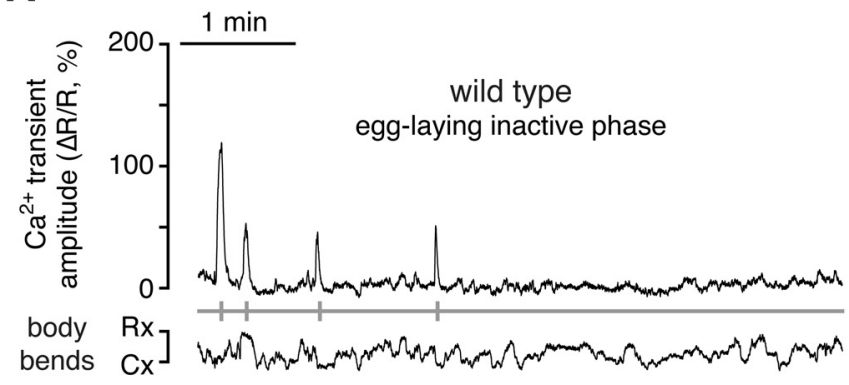

C

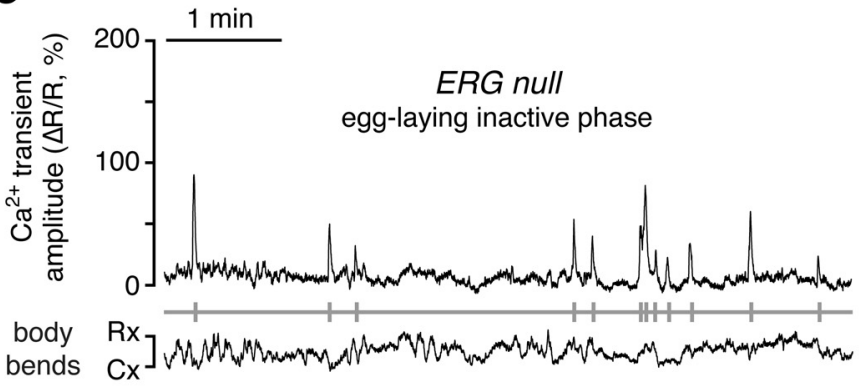

B

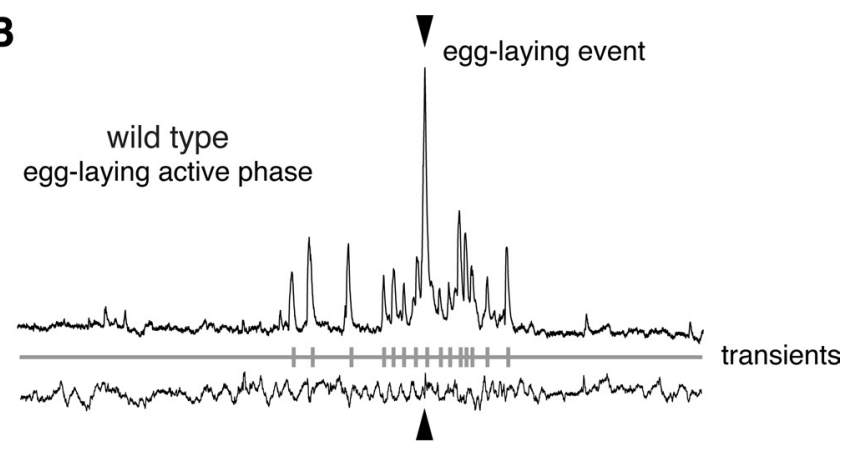

D

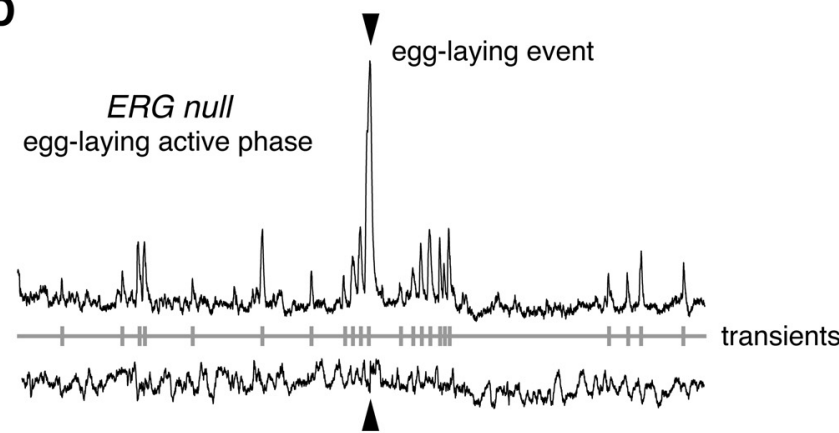

$\mathbf{E}$

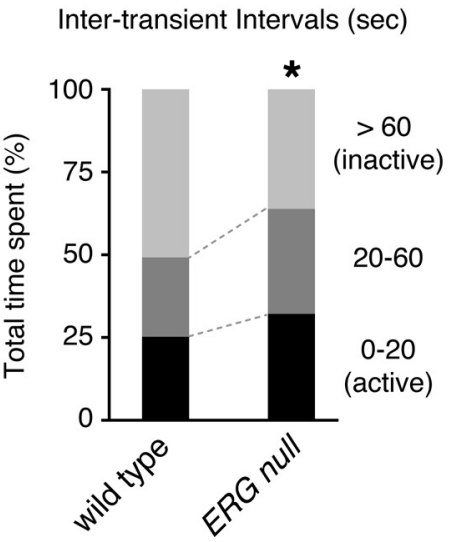

Figure 8. Increased frequency of rhythmic $\mathrm{Ca}^{2+}$ transients in ERG-null mutants. $\mathbf{A - D}$, Ratiometric $\mathrm{Ca}^{2+}$ imaging in the vulval muscles in behaving animals. Six-minute recordings were made for each of 12 wild-type and ERG-null mutant animals. Representative traces shown indicate the average GCaMP3/mCherry ratio $(\Delta R / R)$ in the vulval muscles of separate wild-type $(\boldsymbol{A}, \boldsymbol{B})$ and ERG-null mutant animals $(\boldsymbol{C}, \boldsymbol{D})$ during an egg-laying inactive phase $(\boldsymbol{A}, \boldsymbol{C})$ or the egg-laying active phase $(\boldsymbol{B}, \boldsymbol{D})$. Transients with peak magnitude $\geq 20 \% \Delta R / R$ (vertical gray lines) were used to define intertransient intervals (below). Traces of the area of the mCherry signal indicate changes in vulval muscle size during body bends. CX, smooth contraction; Rx, smooth relaxation. $\boldsymbol{E}$, Egg-laying muscles of ERG-null mutants have more frequent $\mathrm{Ca}^{2+}$ transients. Transients with $\Delta R / R \geq 20 \%$ were used to define intertransient intervals from 12 wild-type and 12 ERG-null mutant animals. Intervals (wild type, $n=201$; ERG null, $n=240$ ) were grouped by their duration $(0-20 \mathrm{~s}, 20-60 \mathrm{~s}$, and $>60 \mathrm{~s}$ ), and the total time spent within each group is shown as a percentage of total recording time $\left(\sim 72\right.$ min per genotype). Asterisk, $p<0.0001$ ( $\chi^{2}$ test). Error bars, $95 \%$ confidence intervals.

ing of behaving animals to delineate how C. elegans sets excitability of the vulval muscles at the precise levels to allow egg-laying behavior to alternate between inactive and active states. Figure $9 A$ illustrates key features of our results. Rhythmic excitation of vulval muscles at a particular phase of each body bend depolarizes the muscles. Between egg-laying active phases, subthreshold responses typically occur that are too weak to trigger detectable $\mathrm{Ca}^{2+}$ signaling or muscle contraction. During the serotonin-triggered egg-laying active phase, however, the muscles become more excitable so that rhythmic excitation triggers $\mathrm{Ca}^{2+}$ responses that drive twitching and egglaying contractions (Fig. 9A). Loss of postsynaptic ERG $\mathrm{K}^{+}$ channels elevates postsynaptic excitability both between and during egg-laying active phases, increasing the probability of postsynaptic $\mathrm{Ca}^{2+}$ transients that drive twitching and egglaying contractions. Thus the two-state behavior seen in the wild type is degraded to a more continuous pattern of egglaying muscle activity in ERG-null mutants.

Figure $9 B$ models at the cellular and molecular level how activity at the egg-laying synapse is controlled. ACh release from ventral cord motor neurons at a particular phase of each body bend activates vulval muscle nAChR to depolarize the vulval muscle postsynaptic terminus (Waggoner et al., 2000; Kim et al., 2001), accounting for the observed rhythmic excitation of the muscles. Depolarization activates the EGL-19 L-type $\mathrm{Ca}^{2+}$ channels to allow entry of $\mathrm{Ca}^{2+}$ through the plasma membrane and amplify the depolarization. C. elegans lacks voltage-gated $\mathrm{Na}^{+}$ channels and thus uses only $\mathrm{Ca}^{2+}$ channels to generate action potentials (Gao and Zhen, 2011; Liu et al., 2011). ERG, in cooperation with other $\mathrm{K}^{+}$channels, repolarizes the membrane to antagonize the L-type $\mathrm{Ca}^{2+}$ channels to limit postsynaptic $\mathrm{Ca}^{2+}$ signaling so that, during the egg-laying inactive phase, no muscle 

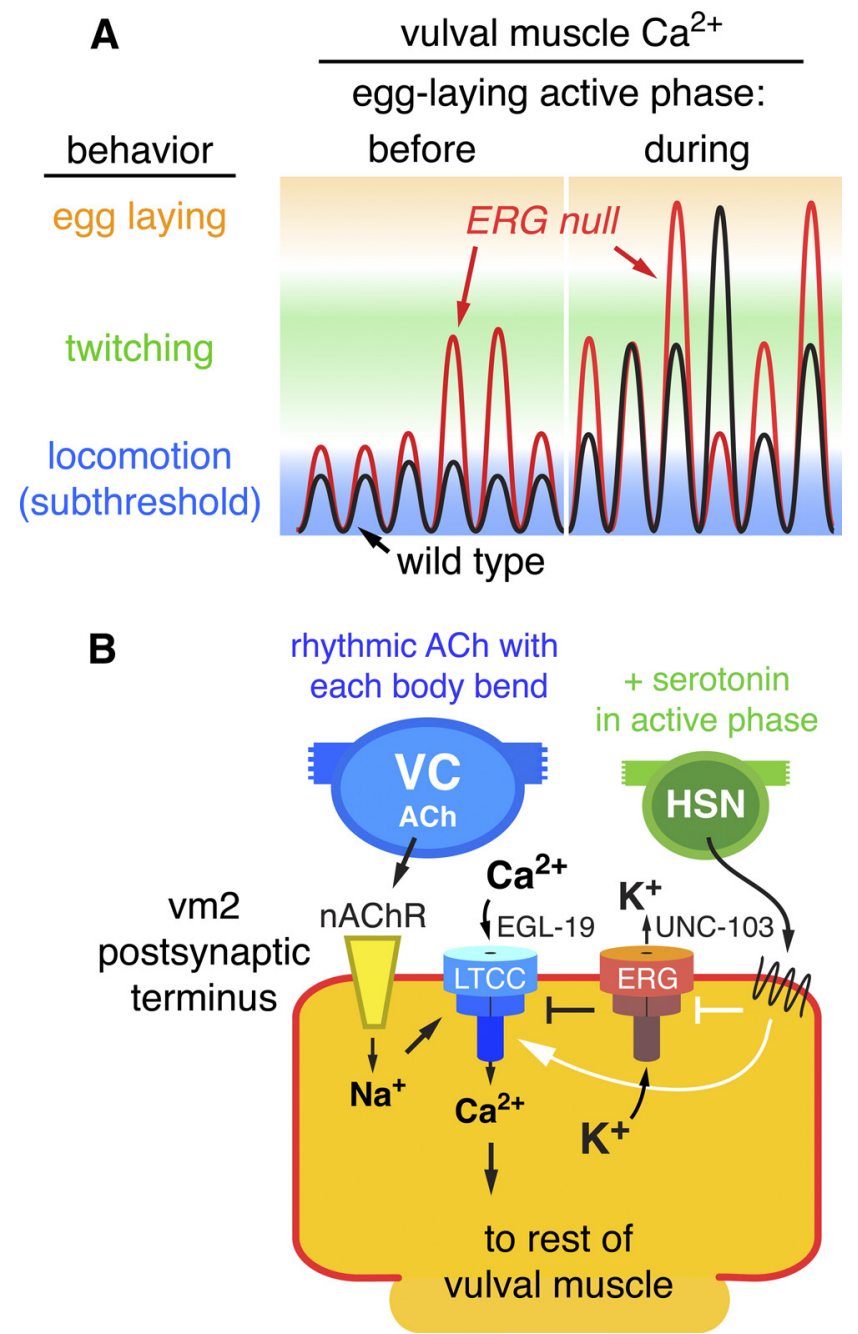

Figure 9. Working model: ERG limits postsynaptic $\mathrm{Ca}^{2+}$ transients and behavioral responses to rhythmic synaptic excitation. $A$, Schematic of vulval muscle $\mathrm{Ca}^{2+}$ signals on a log scale during six body bends before and six more body bends during the active phase of egg laying. Before the active phase, EPSPs are subthreshold and do not trigger detectable vulval muscle $\mathrm{Ca}^{2+}$ transients or contraction. During the active phase, increased postsynaptic electrical excitability increases the probability of $\mathrm{Ca}^{2+}$ transients that drive vulval muscle twitching (green) or large transients that drive complete vulval muscle contraction and egg laying (orange). In the ERG-null mutant (red trace), elevated postsynaptic excitability during both the inactive and active phases increases the probability of superthreshold responses that trigger $\mathrm{Ca}^{2+}$ transients. B, Schematic of control of synaptic excitation at the vulval muscles. ACh release from the VC motor neurons, occurring rhythmically at a particular phase of each locomotor body bend, activates $n A C h R$ on the vm2 postsynaptic terminus. The resulting local membrane depolarization from $\mathrm{Na}^{+}$influx activates EGL-19 voltage-gated L-type $\mathrm{Ca}^{2+}$ channels (LTCC) and plasma membrane $\mathrm{Ca}^{2+}$ entry. UNC-103 ERG K $^{+}$channels localize to and promote repolarization of the postsynaptic terminus to antagonize LTCC activity and keep postsynaptic $\mathrm{Ca}^{2+}$ levels at a subthreshold level that fails to trigger vulval muscle contraction. Loss of ERG leads to sustained $\mathrm{Ca}^{2+}$ entry through LTCC. The HSN motor neuron releases serotonin, which acts via $\mathrm{G}$-protein-coupled receptors to increase vulval muscle response to $\mathrm{ACh}$ and promote the active phase of egg-laying behavior. Solid white arrows and bar indicate possible targets of serotonin signaling: activation of $\mathrm{Ca}^{2+}$ signaling through LTCC and/or inhibition of ERG. Error bars, $95 \%$ confidence intervals.

contraction ensues. The active phase of egg laying is produced by periodic serotonin release from the HSN (Waggoner et al., 1998; Shyn et al., 2003; Zhang et al., 2008). Serotonin activates two vulval muscle serotonin receptors that couple to the $G$ proteins $\mathrm{G} \alpha_{\mathrm{q}}$ and $\mathrm{G} \alpha_{\mathrm{s}}$ (Carnell et al., 2005; Dempsey et al., 2005; Hobson et al., 2006). We observed large $\mathrm{Ca}^{2+}$ responses to the rhythmic excitations that occurred during the active phase. We propose that serotonin signaling initiates the egg-laying active phase by increasing postsynaptic electrical excitability beyond the threshold required to trigger $\mathrm{Ca}^{2+}$ transients and vulval muscle contraction (Fig. 9B). To terminate egg laying, an increase in postsynaptic inhibition, possibly driven by mechanosensation of egg release (Jose et al., 2007), reduces excitability of the vulval muscles. In our model, postsynaptic ERG channels adjust vulval muscle excitability so that during the inactive phase it lies just below the threshold needed to evoke $\mathrm{Ca}^{2+}$ transients and muscle contraction. Release of serotonin and possibly other modulatory factors can then determine when egg laying occurs. We anticipate that ERG functions similarly in neurons that express it to set excitability at thresholds that allow circuits and behaviors to achieve distinct inactive and active states.

Our analysis of postsynaptic excitability in the vulval muscles helps explain how regulation of neurotransmitter release from the presynaptic HSN can effectively control egg laying. Previous work has shown that $\mathrm{G} \alpha_{\mathrm{o}}$ and $\mathrm{G} \alpha_{\mathrm{q}}$ act in the HSN motor neurons to strongly regulate egg laying (Tanis et al., 2008). It is quite possible that much of the regulation of the egg-laying system, for example in response to sensory stimulation, occurs by controlling the timing and extent of serotonin release from the HSN (Ringstad and Horvitz, 2008; Zhang et al., 2008).

Our work illuminates the results of previous studies of $C$. elegans egg-laying behavior. First, our observations of increased twitching and increased $\mathrm{Ca}^{2+}$ transients during the egg-laying active phase make concrete the existence of an active phase with increased vulval muscle excitability. This was previously hypothesized from the indirect evidence that egg-laying events are clustered in time (Waggoner et al., 1998) and the effects of exogenous serotonin on vulval muscle $\mathrm{Ca}^{2+}$ signaling (Shyn et al., 2003). Statistical analyses also suggested there was an underlying rhythmic pattern activating egg-laying events during the active phase, but the nature of the pattern generator remained unknown (Waggoner et al., 1998). Our work suggests that the pattern generator is or is coupled to the periodic body bends of locomotion. A previous study determined that serotonin released from the HSN stimulates a sharp increase in animal locomotion about $30 \mathrm{~s}$ before the onset of an active phase (Hardaker et al., 2001). The purpose for which the HSN simultaneously activates both body bends and the vulval muscles remained the object of speculation. Our work suggests that locomotor body bends promote the rhythmic stimulation that triggers egg laying, and that it therefore makes sense for the worm to activate locomotion at the same time it turns up excitability of the vulval muscles to effectively generate egg-laying behavior.

How do body bends result in rhythmic excitation of the vulval muscles? One obvious potential source of rhythmic input to the vulval muscles is the ventral nerve cord motor neurons. The cholinergic VA and VB motor neurons that direct body-wall muscle contraction during body bends also make synapses consisting of single active zones onto the vm 1 vulval muscles (White et al., 1986). However vulval muscle $\mathrm{Ca}^{2+}$ transients, twitches, and egg-laying events are initiated out of phase with contraction of the adjacent body-wall muscles, arguing against the functional significance of these anatomically minor synapses. Far more extensive synapses onto the vulval muscles come from the six VC motor neurons. The VC neurons show rhythmic $\mathrm{Ca}^{2+}$ transients during locomotion (Faumont et al., 2011), but they only receive significant synaptic input from each other and the HSNs (White et al., 1986). While VC4 and VC5 project short varicosities that synapse onto the vm2 lateral projections (Fig. 1), VC1-3 and VC6 have long, anterior-directed and posterior-directed processes 
that synapse with each other along the body and with VC4/5 at the vulva (Bany et al., 2003). Twitches and egg laying typically start when VC1-VC3 enter the ventral contracted phase (see Fig. $1 A$, bottom). We propose that the VC neurons provide postural, proprioceptive feedback to rhythmically excite the vulval muscles phased with body bends. In this role, the VCs would function like the DVA neuron, which extends processes along the ventral cord, mechanically senses body bends, and uses this information to modulate amplitude of the locomotion waveform (Li et al., 2006; $\mathrm{Hu}$ et al., 2011). The purpose of activating the vulval muscles in advance of ventral contraction is not clear, but it may be mechanical. When the adjacent body-wall muscles are relaxed, the vulva has more room to open and eggs can be more easily released. The rearward wave of body-wall muscle contraction may provide additional peristaltic compression to the uterus and promote egg positioning near the vulva. In an analogous mechanism, a wave of body-wall muscle contraction pushes intestinal contents posteriorly just before anal depressor muscle contraction during the defecation motor program (Liu and Thomas, 1994).

Humans and C. elegans each have $>70$ kinds of $\mathrm{K}^{+}$channels that inhibit cell electrical excitability. An overarching issue is to understand for what purpose complex mixtures of $\mathrm{K}^{+}$channels that differ in individual cell types limit cell excitability, and how and why neuromodulators then further adjust cell excitability. Our studies have delineated a physiological role for the ERG $\mathrm{K}^{+}$ channel in limiting excitability of the vulval muscles so that contractions do not occur until an egg-laying active phase is initiated by serotonin. ERG is only one of several $\mathrm{K}^{+}$channels known to be expressed in the vulval muscles and/or to affect egg laying. The others include EAG, $\mathrm{Kv} 3 /$ Shaw, and $\mathrm{Ca}^{2+}$-activated $\mathrm{K}^{+}$channels (Elkes et al., 1997; Johnstone et al., 1997; Weinshenker et al., 1999; Wang et al., 2001). Gain-of-function mutations in several of these channels inhibit egg laying, but it is less clear if or how loss of these channels affects egg laying. The methods we have developed to successfully delineate how ERG affects egg laying can now be applied to analyzing other $\mathrm{K}^{+}$channel mutants and to the many other existing C. elegans mutants with decreased or increased egg-laying behavior. Together, this approach should help define at a new level of detail how excitability of the vulval muscles is controlled to generate the two behavioral states of egg laying. In particular, the manner in which serotonin signals through $G$ proteins to increase excitability of the vulval muscles to switch from the inactive to the active phase is open to such analysis.

\section{References}

Bany IA, Dong MQ, Koelle MR (2003) Genetic and cellular basis for acetylcholine inhibition of Caenorhabditis elegans egg-laying behavior. J Neurosci 23:8060-8069. Medline

Bellemer A, Hirata T, Romero MF, Koelle MR (2011) Two types of chloride transporters are required for $\mathrm{GABA}(\mathrm{A})$ receptor-mediated inhibition in C. elegans. EMBO J 30:1852-1863. CrossRef Medline

Brenner S (1974) The genetics of Caenorhabditis elegans. Genetics 77:71-94. Medline

Carnell L, Illi J, Hong SW, McIntire SL (2005) The G-protein-coupled serotonin receptor SER-1 regulates egg laying and male mating behaviors in Caenorhabditis elegans. J Neurosci 25:10671-10681. CrossRef Medline

Chase DL, Koelle MR (2004) Genetic analysis of RGS protein function in Caenorhabditis elegans. Meth Enzymol 389:305-320. CrossRef Medline

Clark SG, Lu X, Horvitz HR (1994) The Caenorhabditis elegans locus lin-15, a negative regulator of a tyrosine kinase signaling pathway, encodes two different proteins. Genetics 137:987-997. Medline

Dempsey CM, Mackenzie SM, Gargus A, Blanco G, Sze JY (2005) Serotonin (5HT), fluoxetine, imipramine and dopamine target distinct $5 \mathrm{HT}$ receptor signaling to modulate Caenorhabditis elegans egg-laying behavior. Genetics 169:1425-1436. Medline
Desai C, Garriga G, McIntire SL, Horvitz HR (1988) A genetic pathway for the development of the Caenorhabditis elegans HSN motor neurons. Nature 336:638-646. CrossRef Medline

Dong MQ, Chase D, Patikoglou GA, Koelle MR (2000) Multiple RGS proteins alter neural $G$ protein signaling to allow C. elegans to rapidly change behavior when fed. Genes Dev 14:2003-2014. Medline

Duerr JS, Gaskin J, Rand JB (2001) Identified neurons in C. elegans coexpress vesicular transporters for acetylcholine and monoamines. Am J Physiol Cell Physiol 280:C1616-C1622. Medline

Edwards SL, Charlie NK, Milfort MC, Brown BS, Gravlin CN, Knecht JE, Miller KG (2008) A novel molecular solution for ultraviolet light detection in Caenorhabditis elegans. PLoS Biol 6:e198. CrossRef Medline

Elkes DA, Cardozo DL, Madison J, Kaplan JM (1997) EGL-36 Shaw channels regulate C. elegans egg-laying muscle activity. Neuron 19:165-174. CrossRef Medline

Faumont S, Rondeau G, Thiele TR, Lawton KJ, McCormick KE, Sottile M, Griesbeck O, Heckscher ES, Roberts WM, Doe CQ, Lockery SR (2011) An image-free opto-mechanical system for creating virtual environments and imaging neuronal activity in freely moving Caenorhabditis elegans. PLoS ONE 6:e24666. CrossRef Medline

Frøkjaer-Jensen C, Davis MW, Hopkins CE, Newman BJ, Thummel JM, Olesen SP, Grunnet M, Jorgensen EM (2008) Single-copy insertion of transgenes in Caenorhabditis elegans. Nat Genet 40:1375-1383. CrossRef Medline

Gao S, Zhen M (2011) Action potentials drive body wall muscle contractions in Caenorhabditis elegans. Proc Natl Acad Sci U S A 108:2557-2562. CrossRef Medline

Garcia LR, Sternberg PW (2003) Caenorhabditis elegans UNC-103 ERG-like potassium channel regulates contractile behaviors of sex muscles in males before and during mating. J Neurosci 23:2696-2705. Medline

Hapiak VM, Hobson RJ, Hughes L, Smith K, Harris G, Condon C, Komuniecki P, Komuniecki RW (2009) Dual excitatory and inhibitory serotonergic inputs modulate egg laying in Caenorhabditis elegans. Genetics 181:153-163. Medline

Hardaker LA, Singer E, Kerr R, Zhou G, Schafer WR (2001) Serotonin modulates locomotory behavior and coordinates egg-laying and movement in Caenorhabditis elegans. J Neurobiol 49:303-313. CrossRef Medline

Hobson RJ, Hapiak VM, Xiao H, Buehrer KL, Komuniecki PR, Komuniecki RW (2006) SER-7, a Caenorhabditis elegans 5-HT7-like receptor, is essential for the 5-HT stimulation of pharyngeal pumping and egg laying. Genetics 172:159-169. Medline

Hu Z, Pym EC, Babu K, Vashlishan Murray AB, Kaplan JM (2011) A neuropeptide-mediated stretch response links muscle contraction to changes in neurotransmitter release. Neuron 71:92-102. CrossRef Medline

Johnstone DB, Wei A, Butler A, Salkoff L, Thomas JH (1997) Behavioral defects in C. elegans egl-36 mutants result from potassium channels shifted in voltage-dependence of activation. Neuron 19:151-164. CrossRef Medline

Jose AM, Bany IA, Chase DL, Koelle MR (2007) A specific subset of transient receptor potential vanilloid-type channel subunits in Caenorhabditis elegans endocrine cells function as mixed heteromers to promote neurotransmitter release. Genetics 175:93-105. Medline

Kim J, Poole DS, Waggoner LE, Kempf A, Ramirez DS, Treschow PA, Schafer WR (2001) Genes affecting the activity of nicotinic receptors involved in Caenorhabditis elegans egg-laying behavior. Genetics 157:1599-1610. Medline

Kuzhikandathil EV, Oxford GS (2000) Dominant-negative mutants identify a role for GIRK channels in D3 dopamine receptor-mediated regulation of spontaneous secretory activity. J Gen Physiol 115:697-706. CrossRef Medline

LeBoeuf B, Gruninger TR, Garcia LR (2007) Food deprivation attenuates seizures through CaMKII and EAG K ${ }^{+}$channels. PLoS Genet 3:16221632. Medline

Lee RY, Lobel L, Hengartner M, Horvitz HR, Avery L (1997) Mutations in the alphal subunit of an L-type voltage-activated $\mathrm{Ca}^{2+}$ channel cause myotonia in Caenorhabditis elegans. EMBO J 16:6066-6076. CrossRef Medline

Lee T, Luo L (1999) Mosaic analysis with a repressible cell marker for studies of gene function in neuronal morphogenesis. Neuron 22:451-461. CrossRef Medline

Li W, Feng Z, Sternberg PW, Xu XZ (2006) A C. elegans stretch receptor 
neuron revealed by a mechanosensitive TRP channel homologue. Nature 440:684-687. CrossRef Medline

Liu DW, Thomas JH (1994) Regulation of a periodic motor program in C. elegans. J Neurosci 14:1953-1962. Medline

Liu P, Ge Q, Chen B, Salkoff L, Kotlikoff MI, Wang ZW (2011) Genetic dissection of ion currents underlying all-or-none action potentials in $C$. elegans body-wall muscle cells. J Physiol 589:101-117. CrossRef Medline

Lüscher C, Nicoll RA, Malenka RC, Muller D (2000) Synaptic plasticity and dynamic modulation of the postsynaptic membrane. Nat Neurosci 3:545550. CrossRef Medline

Marder E, Bucher D (2007) Understanding circuit dynamics using the stomatogastric nervous system of lobsters and crabs. Annu Rev Physiol 69: 291-316. CrossRef Medline

McDonald TF, Pelzer S, Trautwein W, Pelzer DJ (1994) Regulation and modulation of calcium channels in cardiac, skeletal, and smooth muscle cells. Physiol Rev 74:365-507. Medline

McNally K, Audhya A, Oegema K, McNally FJ (2006) Katanin controls mitotic and meiotic spindle length. J Cell Biol 175:881-891. CrossRef Medline

Mello C, Fire A (1995) DNA transformation. Methods Cell Biol 48: 451-482. CrossRef Medline

Moresco JJ, Koelle MR (2004) Activation of EGL-47, a G $\alpha_{\mathrm{o}}$-coupled receptor, inhibits function of hermaphrodite-specific motor neurons to regulate Caenorhabditis elegans egg-laying behavior. J Neurosci 24:8522-8530. CrossRef Medline

Petersen CI, McFarland TR, Stepanovic SZ, Yang P, Reiner DJ, Hayashi K, George AL, Roden DM, Thomas JH, Balser JR (2004) In vivo identification of genes that modify ether-a-go-go-related gene activity in Caenorhabditis elegans may also affect human cardiac arrhythmia. Proc Natl Acad Sci U S A 101:11773-11778. CrossRef Medline

Reiner DJ, Weinshenker D, Tian H, Thomas JH, Nishiwaki K, Miwa J, Gruninger T, Leboeuf B, Garcia LR (2006) Behavioral genetics of Caenorhabditis elegans unc-103-encoded erg-like $\mathrm{K}(+)$ channel. J Neurogenet 20:41-66. CrossRef Medline

Ringstad N, Horvitz HR (2008) FMRFamide neuropeptides and acetylcholine synergistically inhibit egg-laying by C. elegans. Nat Neurosci 11:1168-1176. CrossRef Medline

Sanguinetti MC, Tristani-Firouzi M (2006) hERG potassium channels and cardiac arrhythmia. Nature 440:463-469. CrossRef Medline

Shyn SI, Kerr R, Schafer WR (2003) Serotonin and Go modulate functional states of neurons and muscles controlling C. elegans egg-laying behavior. Curr Biol 13:1910-1915. CrossRef Medline

Spilker KA, Wang GJ, Tugizova MS, Shen K (2012) C. elegans Muscleblind homolog mbl-1 functions in neurons to regulate synapse formation. Neural Dev 7:7. CrossRef Medline

Tanis JE, Moresco JJ, Lindquist RA, Koelle MR (2008) Regulation of serotonin biosynthesis by the $\mathrm{G}$ proteins Galphao and Galphaq controls serotonin signaling in Caenorhabditis elegans. Genetics 178:157-169. CrossRef Medline

Tanis JE, Bellemer A, Moresco JJ, Forbush B, Koelle MR (2009) The potassium chloride cotransporter KCC-2 coordinates development of inhibitory neurotransmission and synapse structure in Caenorhabditis elegans. J Neurosci 29:9943-9954. CrossRef Medline

Tian L, Hires SA, Mao T, Huber D, Chiappe ME, Chalasani SH, Petreanu L, Akerboom J, McKinney SA, Schreiter ER, Bargmann CI, Jayaraman V, Svoboda K, Looger LL (2009) Imaging neural activity in worms, flies and mice with improved GCaMP calcium indicators. Nat Methods 6:875881. CrossRef Medline

Waggoner LE, Zhou GT, Schafer RW, Schafer WR (1998) Control of alternative behavioral states by serotonin in Caenorhabditis elegans. Neuron 21:203-214. CrossRef Medline

Waggoner LE, Dickinson KA, Poole DS, Tabuse Y, Miwa J, Schafer WR (2000) Long-term nicotine adaptation in Caenorhabditis elegans involves PKC-dependent changes in nicotinic receptor abundance. J Neurosci 20: 8802-8811. Medline

Wang ZW, Saifee O, Nonet ML, Salkoff L (2001) SLO-1 potassium channels control quantal content of neurotransmitter release at the C. elegans neuromuscular junction. Neuron 32:867-881. CrossRef Medline

Weinshenker D, Garriga G, Thomas JH (1995) Genetic and pharmacological analysis of neurotransmitters controlling egg laying in C. elegans. J Neurosci 15:6975-6985. Medline

Weinshenker D, Wei A, Salkoff L, Thomas JH (1999) Block of an ether-ago-go-like $\mathrm{K}(+)$ channel by imipramine rescues egl-2 excitation defects in Caenorhabditis elegans. J Neurosci 19:9831-9840. Medline

White JG, Southgate E, Thomson JN, Brenner S (1986) The structure of the nervous system of the nematode Caenorhabditis elegans. Phil Trans Royal Soc 314:1-340. CrossRef

Zhang M, Chung SH, Fang-Yen C, Craig C, Kerr RA, Suzuki H, Samuel AD, Mazur E, Schafer WR (2008) A self-regulating feed-forward circuit controlling C. elegans egg-laying behavior. Curr Biol 18:1445-1455. CrossRef Medline 